\title{
STABILITY AND ASYMPTOTIC ANALYSIS OF A FLUID-PARTICLE INTERACTION MODEL
}

\author{
JOSÉ A. CARRILLO, THIERRY GOUDON
}

\begin{abstract}
We are interested in coupled microscopic/macroscopic models describing the evolution of particles dispersed in a fluid. The system consists in a Vlasov-Fokker-Planck equation to describe the microscopic motion of the particles coupled to the Euler equations for a compressible fluid. We investigate dissipative quantities, equilibria and their stability properties and the role of external forces. We also study some asymptotic problems, their equilibria and stability and the derivation of macroscopic two-phase models.
\end{abstract}

\section{INTRODUCTION}

Fluid-particle interaction is of primarily importance in sedimentation analysis of disperse suspensions of particles in fluids, one of the issues being the separation of the solid grains from the fluid by external forces: gravity settling processes or centrifugal forces. These procedures find their applications in biotechnology, medicine, waste-water recycling and mineral processing [11]. On the other hand, aerosols and sprays can be also modelled by fluid-particle type interactions in which bubbles of suspended substances are seen as solid particles $[5,6]$. Eventually, such problems also arises in combustion theory, when modelling Diesel engines or rocket propulsors [45, 46].

In what follows, we describe a single specie of disperse particles by a density function $f(t, x, \xi): f(t, x, \xi) \mathrm{d} \xi \mathrm{d} x$ gives the number of particles enclosed at time $t \geq 0$ in the infinitesimal domain of the phase space centered on $(x, \xi) \in \mathbb{R}^{3} \times \mathbb{R}^{3}$, with volume $\mathrm{d} \xi \mathrm{d} x$. A macroscopic description of the dispersed phase is obtained by looking at averages with respect to the $\xi$ variable like the macroscopic density $\int_{\mathbb{R}^{3}} f \mathrm{~d} \xi$, the macroscopic momentum $\int_{\mathbb{R}^{3}} \xi f \mathrm{~d} \xi$ and so on. The surrounding fluid is described by its density $n(t, x) \geq 0$ and its velocity field $u(t, x) \in \mathbb{R}^{3}$. In this work, we will consider the fluid as compressible and we will describe it by the compressible Euler

2000 Mathematics Subject Classification. 35Q99 35B25.

Key words and phrases. Fluid-Particles Interaction. Vlasov-Euler system. Stability. Hydrodynamic Limit. 
equations. The coupled system of the kinetic transport equation for the particles and the compressible Euler equation for the fluid will be referred as the Vlasov-Euler system.

Throughout the paper, particles are assumed spherically shaped with constant radius $a>0$, so that the mass of a particle is given by $m_{\mathrm{P}}=$ $\frac{4}{3} \rho_{\mathrm{P}} \pi a^{3}, \rho_{\mathrm{P}}$ being the particle mass per unit volume. The evolution of the two phases is coupled through friction force terms. Indeed, the surrounding fluid produces a friction force on the particle, which is proportional to the relative velocity

$$
F(t, x, \xi)=6 \pi \mu a(u(t, x)-\xi),
$$

with $\mu>0$ being the (dynamic) viscosity of the fluid. Accordingly, the force exerted by the particles on the fluid is given by the sum

$$
6 \pi \mu a \int_{\mathbb{R}^{3}}(\xi-u(t, x)) f \mathrm{~d} \xi
$$

Furthermore, both phases are affected by the external forces, which are supposed to derive from a time independent potential $\Phi(x)$. Precisely, we denote by $-m_{\mathrm{P}} \nabla_{x} \Phi$ the force exerted on the particles, and $\alpha \rho_{\mathrm{F}} \nabla_{x} \Phi$ the force exerted per unit volume on the fluid, where $\rho_{\mathrm{F}}$ is the fluid mass per unit volume and $\alpha \in \mathbb{R}$ is a dimensionless parameter (which measures the ratio of the strength of the external force on each phase). External forces can be produced by a great deal of relevant physical mechanisms: gravity, centrifugal, electric or magnetic forces on charged particles/fluid... Throughout the paper, we assume that the potential $\Phi$ does not depend on the unknowns $f, n, u$. It is worthwhile to have in mind the simplest example of settling gravity processes. Here, the force exerted on each particle is the sum of the weight and the buoyancy force:

$$
-\frac{4}{3} \rho_{\mathrm{P}} \pi a^{3} g\left(1-\frac{\rho_{\mathrm{F}}}{\rho_{\mathrm{P}}}\right) e_{3},
$$

where $g$ is the gravity acceleration and $e_{3}$ the upwards unit vector. Therefore, in such a case we have

$$
\Phi(x)=\left(1-\rho_{\mathrm{F}} / \rho_{\mathrm{P}}\right) g x_{3} \quad \alpha=\frac{1}{1-\rho_{\mathrm{F}} / \rho_{\mathrm{P}}} .
$$

Note in particular that the sign of $\Phi$ depends on the ratio of the densities - Another example is given by considering a centrifugal force: $\Phi(x)=$ $\int_{|x|}^{\infty} \varphi(z) \mathrm{d} z \geq 0$, for some function $\varphi: \mathbb{R}^{+} \rightarrow \mathbb{R}^{+}$which tends to 0 at infinity.

Finally, the particles are also subject to Brownian motion, which translates into diffusion in the variable $\xi$, the diffusion coefficient being defined 
by the Einstein formula [25]

$$
\frac{k \theta_{0}}{m_{\mathrm{P}}} \frac{6 \pi \mu a}{m_{\mathrm{P}}}=\frac{k \theta_{0}}{m_{\mathrm{P}}} \frac{9 \mu}{2 a^{2} \rho_{\mathrm{P}}} .
$$

Here $k$ stands for the Boltzmann constant, and $\theta_{0}>0$ denotes the temperature of the surrounding fluid, assumed to be constant. Therefore, we arrive at the following PDEs system

$(1.1) \partial_{t} f+\xi \cdot \nabla_{x} f-\nabla_{x} \Phi \cdot \nabla_{\xi} f=\frac{9 \mu}{2 a^{2} \rho_{\mathrm{P}}} \operatorname{div}_{\xi}\left((\xi-u) f+\frac{k \theta_{0}}{m_{\mathrm{P}}} \nabla_{\xi} f\right)$,

$$
\partial_{t} n+\operatorname{div}_{x}(n u)=0,
$$

$$
\rho_{\mathrm{F}}\left(\partial_{t}(n u)+\operatorname{Div}_{x}(n u \otimes u)+\alpha n \nabla_{x} \Phi\right)+\nabla_{x} p(n)=6 \pi \mu a \int_{\mathbb{R}^{3}}(\xi-u) f \mathrm{~d} \xi .
$$

In (1.3), $p(n)$ is a general pressure law, for instance $p(n)=C_{\gamma} n^{\gamma}, \gamma \geq 1$, $C_{\gamma}>0$. The system is completed by the initial condition

$$
f_{0} \geq 0, \quad n_{0} \geq 0, \quad u_{0} \in \mathbb{R}^{3} .
$$

Besides, if fluid and particles evolve in a domain $\Omega \subset \mathbb{R}^{3}$, we should also add boundary conditions. Boundary conditions will be considered in such a way that the total number of particles and mass of the fluid remain constant on the evolution. The precise boundary conditions are discussed in Section 3.

Although the fluid has been described by the compressible Euler equations, the fluid viscosity plays a major role in the definition of the friction forces. This is completely coherent since in a lot of practical situations viscosity effects are negligible on the fluid evolution. For instance, such a Vlasov-Euler description was introduced for reacting flows of sprays in $[45,46]$ and it is used in Los Alamos National Laboratory by O'Rourke and collaborators [41, 1, 2] to derive the code KIVA-II, as well as in the French Atomic Commission, [5]. This remark will be completely clarified once we choose relevant dimensionless parameters.

Coupled kinetic-fluid model have been introduced in [13]. Derivation of such coupled micro/macro systems are discussed with various viewpoints in $[26,33,36]$. Mathematical analysis of a system coupling the Vlasov equation (1.1) to the constant density incompressible Navier-Stokes equation can be found in [31], while hydrodynamic limits for this model are considered in [28, 29], following the preliminary work [32]. Similar singular perturbations problems are dealt with in $[10,22,23,24,27,35]$. Existence of a smooth solution to the Cauchy problem (1.1), (1.2), (1.3) on a small enough interval of time, is discussed in [5]. 
We wish to address some stability and asymptotic limit questions for the problem (1.1)-(1.3). To this end, it is more convenient to work on the dimensionless version of (1.1)-(1.3). This is discussed in Section 2. The cornerstone of our analysis relies on a dissipation property of the system (1.1)-(1.3), which is presented in Section 3. In turn, this leads to the definition of (non trivial) equilibrium states which can be shown to be nonlinearly stable. Then, we can also guess on formal grounds the asymptotic behavior of the system with respect to the evolution of the physical parameters of the problem. In the spirit of hydrodynamic limits, we obtain coupled two-phase macroscopic equations. This is explained in Section 4. However a complete and rigorous proof of convergence for this problem is far beyond the scope of this paper. Instead, we refer to the progresses obtained in [40]. In Section 5 we explore the limiting two-phase systems and we show the dissipation of a quantity that implies again the nonlinear stability of non trivial equilibrium states. In the gravity settling case these equilibrium states correspond to sedimentation profiles. Despite of the lack of a rigorous proof of convergence in the studied asymptotic limits, we bring out the relevancy of our analysis by studying rigorously the linearized version of (1.1)-(1.3) in the Section 6 .

\section{Modelling Issues}

2.1. Dimensionless System. Let us first remark that

$$
\mathcal{T}_{S}=\frac{m_{\mathrm{P}}}{6 \pi \mu a}=\frac{2 \rho_{\mathrm{P}} a^{2}}{9 \mu}
$$

is the natural relaxation time for the kinetic equation (1.1), usually referred in these applications as the Stokes settling time. We consider as usual

$$
\mathcal{V}_{t h}=\sqrt{\frac{k \theta_{0}}{m_{\mathrm{P}}}}
$$

as the measure of the fluctuation of particles velocity, called their thermal speed.

Let us introduce time and length units $\mathrm{T}$ and $\mathrm{L}$ respectively, which define the time and length units of the observation. We associate a velocity unit $\mathrm{U}=\mathrm{L} / \mathrm{T}$. Then, we can define dimensionless variables. Adopting the convention that primed quantities are dimensionless, we set

$$
\begin{array}{ll}
t=\mathrm{T} t^{\prime}, & x=\mathrm{L} x^{\prime}, \\
n\left(\mathrm{~T} t^{\prime}, \mathrm{L} x^{\prime}\right)=n^{\prime}\left(t^{\prime}, x^{\prime}\right) & u\left(\mathrm{~T} t^{\prime}, \mathrm{L} x^{\prime}\right)=\mathrm{U} u^{\prime}\left(t^{\prime}, x^{\prime}\right)
\end{array} \quad \xi=\mathcal{V}_{t h} \xi^{\prime}
$$

where $\mathcal{P}$ is a pressure unit, and

$$
f^{\prime}\left(t^{\prime}, x^{\prime}, \xi^{\prime}\right)=\frac{4}{3} \pi a^{3} \mathcal{V}_{t h}^{3} f\left(\mathrm{~T} t^{\prime}, \mathrm{L} x^{\prime}, \mathcal{V}_{t h} \xi^{\prime}\right)
$$


Note that $\rho_{\mathrm{F}} n \mathrm{~d} x$ gives the mass of fluid enclosed in a domain with volume $\mathrm{d} x$, so that $n$ is already dimensionless. We eventually associate to the external potential a velocity $\mathcal{V}_{S}$ by setting

$$
\Phi\left(\mathrm{T} t^{\prime}, \mathrm{L} x^{\prime}\right)=\frac{\mathcal{V}_{S} \mathrm{~L}}{\mathcal{T}_{S}} P h i^{\prime}\left(t^{\prime}, x^{\prime}\right) .
$$

Therefore, (1.1) can be recast as

$$
\begin{gathered}
\frac{1}{\mathrm{~T}} \partial_{t^{\prime}} f^{\prime}+\frac{\mathcal{V}_{t h}}{\mathrm{~L}} \xi^{\prime} \cdot \nabla_{x^{\prime}} f^{\prime}-\frac{\mathcal{V}_{S}}{\mathcal{T}_{S} \mathcal{V}_{t h}} \nabla_{x^{\prime}} \Phi^{\prime} \cdot \nabla_{\xi^{\prime}} f^{\prime}= \\
=\frac{1}{\mathcal{T}_{S} \mathcal{V}_{t h}} \operatorname{div}_{\xi^{\prime}}\left(\left(\mathcal{V}_{t h} \xi^{\prime}-\mathrm{U} u^{\prime}\right) f^{\prime}+\mathcal{V}_{t h} \nabla_{\xi^{\prime}} f^{\prime}\right),
\end{gathered}
$$

while (1.2) and (1.3) become

$$
\begin{gathered}
\frac{1}{\mathrm{~T}} \partial_{t^{\prime}} n^{\prime}+\frac{\mathrm{U}}{\mathrm{L}} \operatorname{div}_{x^{\prime}}\left(n^{\prime} u^{\prime}\right)=0, \\
\frac{\mathrm{U}}{\mathrm{T}} \partial_{t^{\prime}}\left(n^{\prime} u^{\prime}\right)+\frac{\mathrm{U}^{2}}{\mathrm{~L}} \operatorname{Div}_{x^{\prime}}\left(n^{\prime} u^{\prime} \otimes u^{\prime}\right)+\frac{\mathcal{P}}{\rho_{\mathrm{F}} \mathrm{L}} \nabla_{x^{\prime}} p^{\prime}\left(n^{\prime}\right)+\alpha \frac{\mathcal{V}_{S}}{\mathcal{T}_{S}} n^{\prime} \nabla_{x^{\prime}} \Phi^{\prime} \\
=\frac{1}{\mathcal{T}_{S}} \frac{\rho_{\mathrm{P}}}{\rho_{\mathrm{F}}} \int_{\mathbb{R}^{3}}\left(\mathcal{V}_{t h} \xi^{\prime}-\mathrm{U} u^{\prime}\right) f^{\prime} \mathrm{d} \xi^{\prime} .
\end{gathered}
$$

Let us define the following dimensionless quantities

$$
\begin{cases}\beta=\frac{\mathrm{T}}{\mathrm{L}} \mathcal{V}_{t h}=\frac{\mathcal{V}_{t h}}{\mathrm{U}}, & \frac{1}{\epsilon}=\frac{\mathrm{T}}{\mathcal{T}_{S}}, \\ \eta=\frac{\mathcal{V}_{S} \mathrm{~T}}{\mathcal{V}_{t h} \mathcal{T}_{S}}, & \chi=\frac{\mathcal{P T}}{\rho_{\mathrm{F}} \mathrm{LU}}=\operatorname{frac} \mathcal{P} \rho_{\mathrm{F}} \mathrm{U}^{2} .\end{cases}
$$

Hence, dropping the primes we are led to

$$
\left\{\begin{array}{l}
\partial_{t} f+\beta \xi \cdot \nabla_{x} f-\eta \nabla_{x} \Phi \cdot \nabla_{\xi} f=\frac{1}{\epsilon} \operatorname{div}_{\xi}\left(\left(\xi-\frac{1}{\beta} u\right) f+\nabla_{\xi} f\right), \\
\partial_{t} n+\operatorname{div}_{x}(n u)=0, \\
\partial_{t}(n u)+\operatorname{Div}_{x}(n u \otimes u)+\chi \nabla_{x} p(n)+\alpha \beta \eta n \nabla_{x} \Phi=\frac{1}{\epsilon} \frac{\rho_{\mathrm{P}}}{\rho_{\mathrm{F}}}(J-\rho u),
\end{array}\right.
$$

where we use the notation

$$
\rho(t, x)=\int_{\mathbb{R}^{3}} f(t, x, \xi) \mathrm{d} \xi, \quad J(t, x)=\beta \int_{\mathbb{R}^{3}} \xi f(t, x, \xi) \mathrm{d} \xi .
$$

For further purposes, it is also convenient to define the kinetic pressure

$$
\mathbb{P}(t, x)=\int_{\mathbb{R}^{3}} \xi \otimes \xi f(t, x, \xi) \mathrm{d} \xi .
$$


Remark 1. Let us go back to the example of the gravity to explain in this particular case the meaning of $\mathcal{V}_{S}$. Consider a particle with radius a and mass per unit volume $\rho_{\mathrm{P}}$. The particle is dropped in a fluid at rest, having viscosity $\mu$ and mass per unit volume $\rho_{\mathrm{F}}$. Hence, the motion of the particle is simply described by the $O D E$

$$
\frac{4}{3} \pi \rho_{\mathrm{P}} a^{3} \frac{\mathrm{d}^{2}}{\mathrm{~d} t^{2}} X(t)=-6 \pi \mu a \frac{\mathrm{d}}{\mathrm{d} t} X(t)-\frac{4}{3} \pi \rho_{\mathrm{P}} a^{3} g\left(1-\frac{\rho_{\mathrm{F}}}{\rho_{\mathrm{P}}}\right) .
$$

Then, as time becomes large, the velocity of the particle $\frac{\mathrm{d}}{\mathrm{d} t} X(t)$ has a limit, the modulus of which is

$$
\mathcal{V}_{S}=\mathcal{T}_{S} g\left|1-\frac{\rho_{\mathrm{F}}}{\rho_{\mathrm{P}}}\right| .
$$

This is referred as the Stokes settling velocity.

Remark 2. Still for the example of gravity, the dimensionless coefficient $\alpha \beta \eta$ is nothing but the inverse of the Froude number of the flow. Accordingly, $1 / \eta$ can be seen as the (reduced) Froude number of the dispersed phase.

Remark 3. We have mentioned in the Introduction that it could be strange to neglect the viscosity term $\mu \Delta_{x} u$ in the fluid equation, while viscosity plays a crucial role in the definition of the friction force. However, when writing the equation in dimensionless form, the corresponding diffusion coefficient reads

$$
\frac{2}{9}\left(\frac{a}{\mathrm{~L}}\right)^{2} \frac{\mathrm{T}}{\mathcal{T}_{S}} \frac{\rho_{\mathrm{P}}}{\rho_{\mathrm{F}}} .
$$

Hence, we realize that this term is negligible under the natural modelling assumption $a \ll \mathrm{L}$, whatever will be our scaling assumptions on $\rho_{\mathrm{P}} / \rho_{\mathrm{F}}$ and $\mathrm{T} / \mathcal{T}_{S}$.

2.2. Boundary Conditions. From now on, we consider the problem (2.2) with the space variable $x \in \Omega \subset \mathbb{R}^{3}$, with either $\Omega=\mathbb{R}^{3}$ itself or a bounded domain with smooth boundary. In such a case we denote by $\nu(x)$ the outward unit vector at $x \in \partial \Omega$. Then, the problem has to be completed with boundary conditions. For the fluid, it is natural to require

$$
u \cdot \nu(x)=0, \quad \text { for } x \in \partial \Omega
$$

for solid boundaries. For the particles, we consider a general boundary condition which describes how particles are reflected by the boundary. Let us denote by $f_{ \pm}(t, x, \xi)$ the trace of $f$ on the set

$$
\Sigma_{ \pm}=\left\{(t, x, \xi) \in \mathbb{R}^{+} \times \partial \Omega \times \mathbb{R}^{3}, \pm \xi \cdot \nu(x) \geq 0\right\} .
$$

Details on the theory of traces for transport equations can be found in e.g. to $[8,16,17,19]$. The boundary condition relates the incoming trace to the 
outgoing one as follows

$$
\begin{aligned}
|\xi \cdot \nu(x)| f_{-}(t, x, \xi)= & \int_{\xi^{\prime} \cdot \nu(x)>0} K\left(x, \xi, \xi^{\prime}\right) f_{+}\left(t, x, \xi^{\prime}\right) \xi^{\prime} \cdot \nu(x) \mathrm{d} \xi^{\prime} \\
& \text { for }(t, x, \xi) \in \Sigma_{-}
\end{aligned}
$$

where the kernel $K$ is required to satisfy the following properties

- Non negativeness:

$$
K\left(x, \xi, \xi^{\prime}\right) \geq 0
$$

- Normalization:

$$
\int_{\xi \cdot \nu(x)<0} K\left(x, \xi, \xi^{\prime}\right) \mathrm{d} \xi=1 .
$$

- Preservation of equilibrium:

$$
|\xi \cdot \nu(x)| e^{-|\xi|^{2} / 2}=\int_{\xi^{\prime} \cdot \nu(x)>0} K\left(x, \xi, \xi^{\prime}\right) e^{-\left|\xi^{\prime}\right|^{2} / 2} \xi^{\prime} \cdot \nu(x) \mathrm{d} \xi^{\prime} .
$$

The first condition preserves non negativeness of the solution; the second condition implies the mass conservation: boundaries are neither a source nor a sink of particles and we always have

$$
\frac{\mathrm{d}}{\mathrm{dt}} \int_{\Omega} \int_{\mathbb{R}^{3}} f \mathrm{~d} \xi \mathrm{d} x=0 .
$$

The simplest example is given by the total accomodation reflexion law

$$
|\xi \cdot \nu(x)| f_{-}(t, x, \xi)=|\xi \cdot \nu(x)| e^{-|\xi|^{2} / 2} \frac{1}{2 \pi} \int_{\xi^{\prime} \cdot \nu(x)>0} f_{+}\left(t, x, \xi^{\prime}\right) \xi^{\prime} \cdot \nu(x) \mathrm{d} \xi^{\prime},
$$

which means that particles are re-emitted by the wall according to a Gaussian law with a temperature coinciding with the temperature of the surrounding fluid (here 1 in dimensionless variables.)

In some particular cases, periodic boundary conditions are more physically reasonable in certain variables. For instance, in the gravity settling case we might consider the simplest situation of a box $\Omega=[0, L]^{2} \times\left[0, L_{3}\right]$ in which periodic boundary conditions are considered for both $x_{1}, x_{2}$-directions and no-flux boundary conditions of the type described above are imposed in the boundaries on the vertical direction $x_{3}$ since our given potential depends only on the $x_{3}$-variable. Again, with these boundary conditions, particles and fluid total masses are preserved and global boundary fluxes of interest are zero over the variables in which periodic boundary conditions are imposed. 


\section{Entropy Dissipation and Stability}

As mentioned in the Introduction, the system enjoys a remarkable dissipation property. This needs a crucial relation between the scaling parameters, and in turn, it leads to stability properties.

3.1. H-Theorem. Let us assume the following hypotheses on the pressure function $p$ :

(HP1) $p: \mathbb{R}^{+} \rightarrow \mathbb{R}^{+}$is continuous, strictly increasing, satisfies $p(0)=0$ and is of class $\mathrm{C}^{2}$ on $(0,+\infty)$.

(HP2) The enthalpy function $h$, defined by

$$
h(n):=\int_{1}^{n} \frac{p^{\prime}(s)}{s} d s, \quad n \in(0, \infty),
$$

belongs to $\mathrm{L}_{\text {loc }}^{1}(0, \infty)$.

These hypotheses allow us to introduce the internal energy function $\Pi$ associated to the pressure function $p$ by

$$
\Pi:[0, \infty) \rightarrow \mathbb{R}, \quad \Pi(n)=\int_{0}^{n} h(s) d s .
$$

It is well-defined due to (HP2), with $\Pi^{\prime}(n)=h(n)$ and $n \Pi^{\prime \prime}(n)=p^{\prime}(n)$ for all $n \in \mathbb{R}^{+}$and with $\Pi(0)=\Pi^{\prime}(1)=0$.

\section{Remark 4.}

- Considering a power pressure law $p(n)=n^{\gamma}, \gamma>0$, we have $\Pi(n)=$ $\left(n^{\gamma}-\gamma n\right) /(\gamma-1)$ for $\gamma \neq 1$ and $\Pi(n)=n \ln (n)-n$ for $\gamma=1$.

- Standard physical assumptions give pressure-laws functions with exponents $\gamma>1$, and therefore they give rise to degenerate pressure functions at zero in the sense that $p^{\prime}(0)=0$. Nevertheless, we include here the most general case allowing atypical pressure functions. Let us mention that compressible Euler equations with a pressure function like $p(n)=n^{\gamma}, 0<\gamma<1$, were considered in hydrodynamic limits of granular media [9].

Proposition 1. Assuming the scaling

$$
\frac{\rho_{\mathrm{P}}}{\rho_{\mathrm{F}}}=\frac{1}{\beta^{2}}, \quad \eta=\beta,
$$

let us define the free energies associated respectively to the particles and the fluid as follows:

$$
\begin{aligned}
& \mathcal{F}_{\mathrm{P}}(f)=\int_{\Omega} \int_{\mathbb{R}^{3}}\left(f \ln (f)+\frac{\xi^{2}}{2} f+\Phi f\right) \mathrm{d} \xi \mathrm{d} x \\
& \mathcal{F}_{\mathrm{F}}(n, u)=\int_{\Omega}\left(n \frac{|u|^{2}}{2}+\chi \Pi(n)+\alpha \beta \eta \Phi n\right) \mathrm{d} x .
\end{aligned}
$$


Then the total free energy functional, $\mathcal{F}(f, n, u)=\mathcal{F}_{\mathrm{P}}(f)+\mathcal{F}_{\mathrm{F}}(n, u)$, is dissipated along the flow, precisely

$$
\frac{\mathrm{d}}{\mathrm{d} t}(\mathcal{F}(f(t), n(t), u(t)))+\frac{1}{\varepsilon} \int_{\Omega} \int_{\mathbb{R}^{3}}\left|\left(v-\beta^{-1} u\right) \sqrt{f}+2 \nabla_{\xi} \sqrt{f}\right|^{2} \mathrm{~d} \xi \mathrm{d} x \leq 0 .
$$

Proof. In the proof below, we perform integration by parts reasoning in the whole space. The slight modifications when dealing with boundaries are detailed at the end. We compute successively the following time derivatives:

- Entropy and kinetic energy of the particles

$$
\begin{aligned}
\frac{\mathrm{d}}{\mathrm{dt}} \int_{\mathbb{R}^{3}} \int_{\mathbb{R}^{3}}\left(f \ln (f)+\frac{\xi^{2}}{2} f\right) \mathrm{d} x i \mathrm{~d} x & =\frac{1}{\varepsilon} \int_{\mathbb{R}^{3}} \int_{\mathbb{R}^{3}}\left(\left(\xi-\beta^{-1} u\right) f+\nabla_{\xi} f\right) \cdot\left(\frac{\nabla_{\xi} f}{f}+\xi\right) \mathrm{d} \xi \mathrm{d} x \\
& \quad-\eta \int_{\mathbb{R}^{3}} \int_{\mathbb{R}^{3}} \nabla_{x} \Phi \cdot \xi f \mathrm{~d} \xi \mathrm{d} x .
\end{aligned}
$$

- Potential energy of the particles

$$
\frac{\mathrm{d}}{\mathrm{dt}} \int_{\mathbb{R}^{3}} \int_{\mathbb{R}^{3}} \Phi f \mathrm{~d} \xi \mathrm{d} x=\beta \int_{\mathbb{R}^{3}} \int_{\mathbb{R}^{3}} \nabla_{x} \Phi \cdot \xi f \mathrm{~d} \xi \mathrm{d} x .
$$

- Kinetic energy of the fluid

$$
\begin{aligned}
\frac{\mathrm{d}}{\mathrm{dt}} \int_{\mathbb{R}^{3}} n \frac{|u|^{2}}{2} \mathrm{~d} x= & -\chi \int_{\mathbb{R}^{3}} u p^{\prime}(n) \cdot \nabla_{x} n \mathrm{~d} x-\alpha \beta \eta \int_{\mathbb{R}^{3}} n u \cdot \nabla_{x} \Phi \mathrm{d} x \\
& +\frac{\beta}{\varepsilon} \frac{\rho_{\mathrm{P}}}{\rho_{\mathrm{F}}} \int_{\mathbb{R}^{3}} \int_{\mathbb{R}^{3}}\left(\xi-\beta^{-1} u\right) f \cdot u \mathrm{~d} \xi \mathrm{d} x .
\end{aligned}
$$

- Entropy of the fluid

$$
\frac{\mathrm{d}}{\mathrm{dt}} \int_{\mathbb{R}^{3}} \Pi(n) \mathrm{d} x=-\int_{\mathbb{R}^{3}} \Pi^{\prime}(n) \operatorname{div}_{x}(n u) \mathrm{d} x=\int_{\mathbb{R}^{3}} \Pi^{\prime \prime}(n) \nabla_{x} n \cdot n u \mathrm{~d} x .
$$

- Potential energy of the fluid

$$
\frac{\mathrm{d}}{\mathrm{dt}} \int_{\mathbb{R}^{3}} n \Phi \mathrm{d} x=\int_{\mathbb{R}^{3}} n u \cdot \nabla_{x} \Phi \mathrm{d} x .
$$

Now, we sum these relations. Taking into account (3.1) and using the fact that $\int_{\mathbb{R}^{3}} u \cdot \nabla_{\xi} f \mathrm{~d} \xi=0$, we arrive at

$$
\begin{aligned}
& \frac{\mathrm{d}}{\mathrm{dt}}(\mathcal{F}(f(t), n(t), u(t)))= \\
& \quad=-\frac{1}{\varepsilon} \int_{\mathbb{R}^{3}} \int_{\mathbb{R}^{3}}\left(\left(\xi-\beta^{-1} u\right)^{2} f+\frac{\left|\nabla_{\xi} f\right|^{2}}{f}\right. \\
&\left.\quad+\left(\xi-\beta^{-1} u\right) f \cdot \frac{\nabla_{\xi} f}{f}+\xi \cdot \nabla_{\xi} f-\beta^{-1} u \cdot \nabla_{\xi} f\right) \mathrm{d} \xi \mathrm{d} x,
\end{aligned}
$$


which ends the proof in the whole space.

When considering boundary conditions, integration by parts yields an additional boundary term, which reads

$$
\int_{\partial \Omega} \int_{\mathbb{R}^{3}}\left(f \ln (f)+\frac{\xi^{2}}{2}+\Phi f\right) \xi \cdot \nu(x) \mathrm{d} \xi \mathrm{d} \sigma(x)
$$

with $\mathrm{d} \sigma(x)$ the Lebesgue measure on $\partial \Omega$. All boundary terms from the fluid equation vanish, by using the physical boundary condition (2.3). The mass conservation property (2.5) satisfied by the kernel $K$ implies that

$$
\int_{\partial \Omega} \Phi(x)\left(\int_{\mathbb{R}^{3}} f \xi \cdot \nu(x) \mathrm{d} \xi\right) \mathrm{d} \sigma(x)=0 .
$$

Then, by combining the conditions $(2.4),(2.5)$ and (2.6), we can check that the remainder term

$$
\int_{\mathbb{R}^{3}}\left(f \ln (f)+\frac{\xi^{2}}{2}\right) \xi \cdot \nu(x) \mathrm{d} \xi=\int_{\mathbb{R}^{3}} f \ln \left(\frac{f}{e^{-\xi^{2} / 2}}\right) \xi \cdot \nu(x) \mathrm{d} \xi
$$

is non positive, as a consequence of the Jensen inequality, a property known as the Darrozès-Guiraud inequality $[18,16]$.

3.2. Relative Entropy. The remarkable fact is that the dissipated quantity can be seen as a relative entropy, which evaluates how far the solution is from an equilibrium state. We refer to [21] for related problems in which similar properties were found. Indeed, let us start with the dispersed phase and set

$$
f_{S}(x, \xi)=Z_{\mathcal{M}_{\mathrm{P}}} e^{-\Phi(x)} M(\xi)
$$

with

$$
M(\xi)=\frac{e^{-\xi^{2} / 2}}{(2 \pi)^{3 / 2}},
$$

and the normalization condition

$$
Z_{\mathcal{M}_{\mathrm{P}}}=\frac{\mathcal{M}_{\mathrm{P}}}{\int_{\Omega} e^{-\Phi(x)} \mathrm{d} x} .
$$

Such a definition makes sense provided $\Phi$ fulfils the confinement condition: (HC1) $x \mapsto e^{-\Phi(x)} \in \mathrm{L}^{1}(\Omega)$.

Then, $f_{S}$ is a (non homogeneous) stationary solution of the kinetic equation in (2.2) with $u=0$, since $\left(\xi \cdot \nabla_{x}-\nabla_{x} \Phi \cdot \nabla_{\xi}\right) f_{S}=0$ as well as 
$\operatorname{div}_{\xi}\left(\xi f_{S}+\nabla_{\xi} f_{S}\right)=0$. When taking into account no-flux boundary conditions, condition (2.6) ensures that $f_{S}$ is still a stationary solution. Since the kinetic equation preserves the total number of particles

$$
\frac{\mathrm{d}}{\mathrm{dt}} \int_{\Omega} \int_{\mathbb{R}^{3}} f \mathrm{~d} \xi \mathrm{d} x=0
$$

we select the equilibrium state $f_{S}$ which has the same total particles mass as the solution of the evolution problem

$$
\mathcal{M}_{\mathrm{P}}=\int_{\Omega} \int_{\mathbb{R}^{3}} f \mathrm{~d} \xi \mathrm{d} x=\int_{\Omega} \int_{\mathbb{R}^{3}} f_{0} \mathrm{~d} \xi \mathrm{d} x=\int_{\Omega} \int_{\mathbb{R}^{3}} f_{S} \mathrm{~d} \xi \mathrm{d} x .
$$

Of course, our equilibrium should be of finite free energy and therefore, we further assume that $\mathcal{F}_{\mathrm{P}}\left(f_{S}\right)<\infty$, which is equivalent to the following confinement condition:

(HC2) $x \mapsto \Phi(x) e^{-\Phi(x)} \in \mathrm{L}^{1}(\Omega)$.

Finally, we remark that

$$
\mathcal{F}_{\mathrm{P}}(f)=\int_{\Omega} \int_{\mathbb{R}^{3}}\left(f \ln \left(\frac{f}{f_{S}}\right)-f+f_{S}\right) \mathrm{d} \xi \mathrm{d} x+\ln \left(\frac{Z_{\mathcal{M}_{\mathrm{P}}}}{(2 \pi)^{3 / 2}}\right) \mathcal{M}_{\mathrm{P}},
$$

and the first term is nonnegative and vanishes if and only if $f=f_{S}$.

More precisely, let us define the relative entropy functional with respect to $f_{S}$ for the particles: $R E_{\mathrm{P}}\left(\cdot \mid f_{S}\right): \mathrm{L}_{+}^{1}\left(\Omega \times \mathbb{R}^{3}\right) \rightarrow \mathbb{R} \cup\{\infty\}$ is given by

$$
R E_{\mathrm{P}}\left(f \mid f_{S}\right)=\left\{\begin{array}{cl}
\int_{\Omega} \int_{\mathbb{R}^{3}}\left(f \ln \left(\frac{f}{f_{S}}\right)-f+f_{S}\right) \mathrm{d} \xi \mathrm{d} x & \text { if } f \ln ^{-} f \in \mathrm{L}^{1}\left(\Omega \times \mathbb{R}^{3}\right), \\
\infty & \text { else, }
\end{array}\right.
$$

where $\mathrm{L}_{+}^{1}\left(\Omega \times \mathbb{R}^{3}\right)=\left\{f \in \mathrm{L}^{1}\left(\Omega \times \mathbb{R}^{3}\right): f \geq 0\right\}$. Here, we follow the notation $g^{-}(x)=-g(x)$ whenever $g(x) \leq 0$ and zero otherwise, analogously for $g^{+}$.

Thus, the free energy of the particles is nothing but the relative entropy with respect to the equilibrium state $f_{S}$ up to a constant, i.e.,

$$
\mathcal{F}_{\mathrm{P}}(f)=R E_{\mathrm{P}}\left(f \mid f_{S}\right)+\ln \left(\frac{Z_{\mathcal{M}_{\mathrm{P}}}}{(2 \pi)^{3 / 2}}\right) \mathcal{M}_{\mathrm{P}} .
$$

Regarding the fluid part of the free energy, we define the functional $E_{\mathrm{F}}$ : $\mathrm{L}_{+}^{1}(\Omega) \rightarrow \mathbb{R} \cup\{\infty\}$

$$
E_{\mathrm{F}}(n)=\left\{\begin{array}{cl}
\int_{\Omega}\left(\frac{\alpha \beta \eta}{\chi} n \Phi+\Pi^{+}(n)\right) d x-\int_{\Omega} \Pi^{-}(n) d x & \text { if } \Pi^{-}(n) \in \mathrm{L}^{1}(\Omega) \\
\infty & \text { else },
\end{array}\right.
$$


where $\mathrm{L}_{+}^{1}(\Omega)=\left\{n \in \mathrm{L}^{1}(\Omega): n \geq 0\right\}$. Restricting this functional to the set of $\mathrm{L}_{+}^{1}(\Omega)$ functions with total fluid mass

$$
\mathcal{M}_{\mathrm{F}}=\int_{\Omega} n \mathrm{~d} x=\int_{\Omega} n_{0} \mathrm{~d} x,
$$

and including this restriction as a Lagrange multiplier for (3.4), we obtain the formal Euler-Lagrange condition, whenever $n_{S}>0$ :

$$
\Pi^{\prime}\left(n_{S}(x)\right)+\frac{\alpha \beta \eta}{\chi} \Phi(x)=Z_{\mathcal{M}_{\mathrm{F}}} \in \mathbb{R}
$$

to be satisfied for a minimizer $n_{S}$ of (3.4) in this set, where $Z_{\mathcal{M}_{\mathrm{F}}}$ is a normalization constant.

The assumptions (HP1)-(HP2) on the pressure function $p$ allow us to define rigorously a stationary state out of the implicit definition (3.5). In fact, $h=\Pi^{\prime}$ is a diffeomorphism from $(0, \infty)$ onto its range $\left(h\left(0^{+}\right), h(\infty)\right)$ and thus, one has to give sense to $n_{S}$ outside the range of $h$. This is done using the generalized inverse $\sigma$ of $h$ defined as

$$
\sigma: \mathbb{R} \rightarrow[0, \infty], \quad \sigma(s)=\left\{\begin{array}{rll}
0 & \text { for } \quad s \leq h(0+), \\
h^{-1}(s) & \text { for } \quad h(0+)<s<h(\infty), \\
\infty & \text { for } \quad h(\infty) \leq s .
\end{array}\right.
$$

Now, we can define our candidates to be minimizers of the functional $E_{\mathrm{F}}(n)$ on the set of $\mathrm{L}_{+}^{1}(\Omega)$ functions with total fluid mass $\mathcal{M}_{\mathrm{F}}$ by

$$
n_{S}(x)=\sigma\left(Z_{\mathcal{M}_{\mathrm{F}}}-\frac{\alpha \beta \eta}{\chi} \Phi(x)\right),
$$

where $Z_{\mathcal{M}_{F}}$ is fixed by imposing the conservation of fluid mass, i.e., $Z_{\mathcal{M}_{F}}$ is implicitly defined by

$$
\mathcal{M}_{\mathrm{F}}=\int_{\Omega} n \mathrm{~d} x=\int_{\Omega} n_{0} \mathrm{~d} x=\int_{\Omega} n_{S} \mathrm{~d} x .
$$

Under the generality of the pressure function (HP1)-(HP2), the fact that the candidate $n_{S}$ is integrable and that there exists a normalization constant $Z_{\mathcal{M}_{\mathrm{F}}}$ achieving the total fluid mass $\mathcal{M}_{\mathrm{F}}$ is not trivial. Therefore, additional confinement assumptions on the potential have to be done in order $n_{S}$ to become a suitable candidate for being a minimizer for $E_{\mathrm{F}}(n)$. These assumptions, the rigorous proof of the fact that $n_{S}$ is a minimizer and the connection of the functional $E_{\mathrm{F}}(n)$ to a suitable notion of relative entropy towards the steady state $n_{S}$ have already been analyzed in complete detail in [14, Subsection 3.1] for general nonlinear diffusions based on ideas developed for the power-law pressure functions in [15]. We will summarize 
the main relevant results. One needs at least a basic regularity assumption on the potential:

(HP3) $\Phi \in \mathrm{W}^{1,1}(\Omega)$ if $\Omega$ is bounded, or $\Phi \in \mathrm{W}_{\text {loc }}^{1,1}(\Omega)$ otherwise.

Further additional confinement conditions on the potential are:

(HC3) $\alpha \Phi$ is a bounded below function on $\Omega$, i.e., there exists a constant $C \in \mathbb{R}$ such that $\alpha \Phi(x) \geq C$ a.e. $x \in \Omega$.

(HC4) $\alpha \Phi$ is coercive on $\Omega$, i.e., $\forall A \in \mathbb{R}:\{x \in \Omega \mid \alpha \Phi(x) \leq A\}$ is bounded.

Due to (HC3) we can assume without loss of generality that the potential $\alpha \Phi(x)$ has zero infimum over $\Omega$ by adding a suitable constant to the potential, which is anyhow defined up to a constant.

Let us consider the one parameter family of functions

$$
n_{Z}(x)=\sigma\left(Z-\frac{\alpha \beta \eta}{\chi} \Phi(x)\right)
$$

with $Z \in \mathbb{R}$ and be $Z^{*}=\sup \left\{Z \in \mathbb{R} \mid n_{Z} \in \mathrm{L}^{1}(\Omega)\right\}$ which is well defined by assuming the additional confinement assumption:

(HC5) There exists $Z \in \mathbb{R}$ such that $n_{Z} \in \mathrm{L}_{+}^{1}(\Omega)$. 
Let us denote by $T M(Z)$ the total mass of $n_{Z}$ in $\Omega$ defined on $\left(-\infty, Z^{*}\right)$. It is obvious that $T M(Z)$ is strictly increasing, and thus, its limit as $Z \nearrow Z^{*}$ is denoted by $\mathcal{M}^{*}$. We will assume that the total fluid mass is in the integrable range:

(HC6) $\mathcal{M}_{\mathrm{F}} \in\left(0, \mathcal{M}^{*}\right)$,

or in other words, that there exists a unique normalization constant $Z_{\mathcal{M}_{\mathrm{F}}}$ such that $n_{S}$ is well defined. Finally, our candidate $n_{S}$ has to have finite free energy and thus, we further assume:

(HC7) $E_{\mathrm{F}}\left(n_{Z}\right)<\infty$ and $\Pi^{-}\left(n_{Z}\right) \in \mathrm{L}^{1}(\Omega)$ for $Z \in\left(-\infty, Z^{*}\right)$.

\section{Remark 5.}

- If $h\left(0^{+}\right)>-\infty$ and $h(\infty)=\infty$, which is the case if $p(n)=n^{\gamma}$, $\gamma>1$, then hypotheses (HC5)-(HC7) are trivially satisfied with $\mathcal{M}^{*}=\infty$. Note also that for $p(n)=n^{\gamma}$, we have

$$
\sigma(s)=\left[\left(\frac{\gamma-1}{\gamma} s+1\right)^{+}\right]^{1 /(\gamma-1)} .
$$

- If $h\left(0^{+}\right)=-\infty$ and $h(\infty)=\infty$, which is the case if $p(n)=n$, then $\mathcal{M}^{*}=\infty$. In case $p(n)=n$, hypotheses $(\mathbf{H C 5})-(\mathbf{H C} 7)$ are equivalent to hypotheses $(\mathbf{H C 1})-(\mathbf{H C 2})$ and $\sigma(s)=e^{s}$.

- In case $\Omega$ is bounded and $\Phi$ is bounded the conditions (HC3)(HC7) are trivially satisfied.

The following result is proven in [14, Proposition 5, Lemma 6]:

Theorem 1. Assuming the conditions (HP1)-(HP3) on the pressure and the potential and the confinement conditions (HC1)-(HC7), then the functional $E_{\mathrm{F}}(n)$ has a unique minimizer given by

$$
n_{S}(x)=\sigma\left(Z_{\mathcal{M}_{\mathrm{F}}}-\frac{\alpha \beta \eta}{\chi} \Phi(x)\right),
$$

in the set of $\mathrm{L}_{+}^{1}(\Omega)$ functions with total fluid mass $\mathcal{M}_{\mathrm{F}}$. Moreover:

$$
E_{\mathrm{F}}(n)-E_{\mathrm{F}}\left(n_{S}\right) \geq \int_{\Omega}\left[\Pi(n)-\Pi\left(n_{S}\right)-\Pi^{\prime}\left(n_{S}\right)\left(n-n_{S}\right)\right](x) d x
$$

with equality if and only if

$$
\frac{\alpha \beta \eta}{\chi} \Phi(x)+h\left(n_{S}(x)\right)=Z_{\mathcal{M}_{\mathrm{F}}}, \quad \text { for almost all } x \in \Omega .
$$

Let us remark that the right-hand side of (3.7) is positive and equal to zero if and only if $n=n_{S}$. Thus, previous theorem allows us to rewrite the fluid free energy functional as

$$
\mathcal{F}_{\mathrm{F}}(n(t), u(t))=\int_{\Omega} n \frac{|u|^{2}}{2} \mathrm{~d} x+\chi\left(E_{\mathrm{F}}(n)-E_{\mathrm{F}}\left(n_{S}\right)\right)+\chi E_{\mathrm{F}}\left(n_{S}\right)
$$


and we observe due to (3.7) that again

$$
R E_{\mathrm{F}}\left((n, u) \mid\left(n_{S}, u_{S}\right)\right)=\int_{\Omega} n \frac{|u|^{2}}{2} \mathrm{~d} x+\chi\left(E_{\mathrm{F}}(n)-E_{\mathrm{F}}\left(n_{S}\right)\right)
$$

is a functional that controls the distance from the pair $(n(t), u(t))$ to the equilibrium solution $\left(n_{S}, u_{S}=0\right)$.

Therefore, the whole free energy functional $\mathcal{F}(f, n, u)$ can be considered, up to a constant, a relative entropy functional towards the equilibrium solution $\left(f_{S}, n_{S}, u_{S}=0\right)$, i.e., defining the relative entropy functional from $(f, n, u)$ to $\left(f_{S}, n_{S}, u_{S}=0\right)$ as

$$
R E\left((f, n, u) \mid\left(f_{S}, n_{S}, u_{S}\right)\right)=R E_{\mathrm{P}}\left(f \mid f_{S}\right)+R E_{\mathrm{F}}\left((n, u) \mid\left(n_{S}, u_{S}\right)\right) .
$$

Thus, we have

$R E\left((f, n, u) \mid\left(f_{S}, n_{S}, u_{S}\right)\right)=\mathcal{F}(f, n, u)-\ln \left(\frac{Z_{\mathcal{M}_{\mathrm{P}}}}{(2 \pi)^{3 / 2}}\right) \mathcal{M}_{\mathrm{P}}-\chi E_{\mathrm{F}}\left(n_{S}\right) \geq 0$, and this quantity vanishes if and only if $f=f_{S}, n=n_{S}$ and $u=u_{S}=0$.

\section{Remark 6.}

- In the gravity settling case and as we discussed before, the physical boundary conditions of interest are periodic boundary conditions in the $x_{1}, x_{2}$ directions and no-flux boundary conditions as (2.3)-(2.6) for the $x_{3}$-direction. All boundary terms in the periodic boundary conditions disappear while boundary terms in the $x_{3}$-direction are treated as above. All conclusions of the last subsections apply equally well to this case.

- For the gravity settling case, in the particular case of $\alpha>0$, which means that gravity dominates over buoyancy force, $f_{S}$ and $n_{S}$ represent the typical sedimentation profiles of particles and fluid respectively. It is interesting to remark that the steady density of dispersed particles will be always positive according to (3.3) while the steady fluid density given by (3.6) might be compactly supported for pressure functions of the form $p(n)=n^{\gamma}, \gamma>1$.

- Let us remark that $\left(f_{S}, n_{S}, u_{S}=0\right)$ is a stationary classical solution of (2.2) wherever $n_{S}$ is regular.

3.3. Nonlinear Stability. It is now standard to obtain stability results from the non increasing character of the relative entropy as was done in $[12,21,42]$ and references therein. In fact, everything is reduced to use the relative entropy functionals to control the distance in $\mathrm{L}^{1}$ or in $\mathrm{L}^{p}$ between the solution $(f(t), n(t), u(t))$ of the system (2.2) and the equilibrium solution $\left(f_{S}, n_{S}, u_{S}\right)$. These inequalities called in the literature Csiszár-KullbackPinsker type inequalities are well-known in the linear case [4] and they are 
already available in the literature [14, Section 4] for the nonlinear case. As a consequence, we obtain directly the following non-linear stability result for the equilibrium solution $\left(f_{S}, n_{S}, u_{S}\right)$.

Theorem 2. Given $(f(t), n(t), u(t))$ a solution to the Cauchy problem for the system $(2.2)$ and $\left(f_{S}, n_{S}, u_{S}=0\right)$ with $f_{S}$ and $n_{S}$ given respectively by (3.3) and (3.6), such that

$$
\int_{\Omega} \int_{\mathbb{R}^{3}} f_{0} \mathrm{~d} \xi \mathrm{d} x=\int_{\Omega} \int_{\mathbb{R}^{3}} f_{S} \mathrm{~d} \xi \mathrm{d} x \quad \text { and } \quad \int_{\Omega} n_{0} \mathrm{~d} x=\int_{\Omega} n_{S} \mathrm{~d} x,
$$

then for any $\epsilon>0$, there exists $\delta>0$ such that if

$$
R E\left(\left(f_{0}, n_{0}, u_{0}\right) \mid\left(f_{S}, n_{S}, u_{S}\right)\right) \leq \delta,
$$

we conclude the solution satisfies

$$
\left\|f(t)-f_{S}\right\|_{\mathrm{L}^{1}\left(\Omega \times \mathbb{R}^{3}\right)} \leq \epsilon,\left\|n(t)-n_{S}\right\|_{\mathrm{L}^{1}(\Omega)} \leq \epsilon \text { and } \int_{\Omega} n(t) \frac{|u(t)|^{2}}{2} \mathrm{~d} x \leq \epsilon
$$

for all $t \geq 0$.

\section{Asymptotic Analysis}

4.1. A priori Estimates and Moments. We are interested in hydrodynamic limits for the problem (2.2), which means that the relaxation time $\mathcal{T}_{S}$ is small compared to the observation time scale $\mathrm{T}$, i.e., we deal with the singular perturbation problem $\varepsilon \rightarrow 0$. We expect that the limit can be described by macroscopic equations, with unknowns that do not depend on the variable $\xi$. Indeed, coming back to the entropy dissipation (3.2), we realize that the system then relaxes to

$$
f(t, x, \xi) \simeq \frac{\rho(t, x)}{(2 \pi)^{3 / 2}} \exp \left(-\frac{\left|\xi-\beta^{-1} u(t, x)\right|^{2}}{2}\right) .
$$

Hence, of course, the limiting behavior highly depends on the behavior of the other scaling parameters.

Since we are interested in hydrodynamic limits, it is convenient to write the macroscopic equations satisfied by the moments of $f$. Integrating the kinetic equation in (2.2) with respect to $\xi$ leads to

$$
\left\{\begin{array}{l}
\partial_{t} \rho+\operatorname{div}_{x} J=0, \\
\partial_{t} J+\beta^{2} \operatorname{Div}_{x} \mathbb{P}+\eta \beta \rho \nabla_{x} \Phi=-\frac{1}{\varepsilon}(J-\rho u) .
\end{array}\right.
$$

Before we specify the behavior of the parameters with respect to $\varepsilon$, let us emphasize the main features of the problem. First, the entropy dissipation can be used to establish useful a priori estimates. 
Proposition 2. Assume that (3.1) holds and that the conditions (HP1)(HP3) on the pressure and the potential and the confinement conditions (HC1)-(HC7) are satisfied. Moreover, we assume further confinement conditions:

(HC8) $\Phi$ is a bounded below function on $\Omega$, i.e., there exists a constant $C \in \mathbb{R}$ such that $\Phi(x) \geq C$ a.e. $x \in \Omega$.

(HC9) $(1+\Phi) \exp (-\nu \Phi(x)) \in \mathrm{L}^{1}(\Omega)$, for some positive constants $C>0$, and $0<\nu<1$.

(HC10) If $h(0+)=-\infty$ we assume there exists $0<s_{1}<1$ such that

$$
\sup \left\{\frac{\Pi(n)}{n h(n)}, 0<n<s_{1}\right\}<+\infty \text {. }
$$

We suppose that the initial data $\left(f_{0}, n_{0}, u_{0}\right)$ satisfies $f_{0} \geq 0, n_{0} \geq 0$ and that the quantities

$$
\begin{aligned}
& \int_{\Omega} \int_{\mathbb{R}^{3}} f_{0}\left(1+\left|\ln \left(f_{0}\right)\right|+\frac{\xi^{2}}{2}+|\Phi|\right) \mathrm{d} \xi \mathrm{d} x \\
& \int_{\Omega}\left(n_{0}+n_{0}\left|u_{0}\right|^{2}+\left|\Pi\left(n_{0}\right)\right|+n_{0} \beta \eta|\alpha \Phi|\right) \mathrm{d} x
\end{aligned}
$$

are finite and bounded uniformly with respect to all the parameters $\varepsilon, \beta, \eta, \alpha, \rho_{\mathrm{P}} / \rho_{\mathrm{F}}$. Then, we have

(i) $f\left(1+\xi^{2}+|\Phi|+|\ln (f)|\right)$ is bounded in $\mathrm{L}^{\infty}\left(\mathbb{R}^{+} ; \mathrm{L}^{1}\left(\Omega \times \mathbb{R}^{3}\right)\right)$.

(ii) $n,|\Pi(n)|$ and $\beta \eta|\alpha \Phi| n$ are bounded in $\mathrm{L}^{\infty}\left(\mathbb{R}^{+} ; \mathrm{L}^{1}(\Omega)\right)$.

(iii) $\sqrt{n} u$ is bounded in $\mathrm{L}^{\infty}\left(\mathbb{R}^{+} ; \mathrm{L}^{2}(\Omega)\right)$.

(iv) $\frac{1}{\sqrt{\varepsilon}}\left(\left(\xi-\beta^{-1} u\right) \sqrt{f}+2 \nabla_{\xi} \sqrt{f}\right)=\frac{D}{\sqrt{\varepsilon}}$ is bounded in $\mathrm{L}^{2}\left(\mathbb{R}^{+} \times \Omega \times \mathbb{R}^{3}\right)$.

In this statement "bounded" means "bounded uniformly with respect to all the parameters $\varepsilon, \beta, \eta, \alpha, \rho_{\mathrm{P}} / \rho_{\mathrm{F}} "$.

Deducing these estimates from the relative entropy is somehow classical. We will give some details of the proof. To this end, we need the following claim.

Lemma 1. Let $\Omega$ be a subset of $\mathbb{R}^{D}$, possibly $\mathbb{R}^{D}$ itself. Let $U: \Omega \rightarrow \mathbb{R}^{+}$ such that $(1+U) e^{-\nu U} \in \mathrm{L}^{1}(\Omega)$ for some $0<\nu<1$. Let $g: \Omega \rightarrow \mathbb{R}^{+}$. Then, we have

$$
0 \leq \int_{\Omega} g \ln ^{-}(g) \mathrm{d} y \leq \nu \int U g \mathrm{~d} y+\int_{\Omega}(1+\nu U) e^{-\nu U} \mathrm{~d} y .
$$


Proof. We follow arguments in [20]. We split

$$
\begin{aligned}
& \int_{0 \leq g \leq 1} g(-\ln (g)) \mathrm{d} y= \\
& =\int_{0 \leq g \leq e^{-\nu U}} g(-\ln (g)) \mathrm{d} y+\int_{e^{-\nu U} \leq g \leq 1} g(-\ln (g)) \mathrm{d} y \\
& \leq e \int_{0 \leq g \leq e^{-\nu U}} \frac{g}{e}\left(-\ln \left(\frac{g}{e}\right)\right) \mathrm{d} y-\int_{0 \leq g \leq e^{-\nu U}} g \ln (e) \mathrm{d} y \\
& \quad+\nu \int_{\nu U \geq-\ln (g) \geq 0} U g \mathrm{~d} y .
\end{aligned}
$$

Then, since $s \mapsto s(-\ln (s))$ is non decreasing on $(0,1 / e)$, we can dominate

$$
e \int_{0 \leq g \leq e^{-\nu U}} \frac{g}{e}\left(-\ln \left(\frac{g}{e}\right)\right) \mathrm{d} y \leq \int_{0 \leq g \leq e^{-\nu U}} e^{-\nu U}\left(-\ln \left(e^{-\nu U}\right)+\ln (e)\right) \mathrm{d} y .
$$

Hence, since $g \geq 0$, we obtain

$$
\int_{0 \leq g \leq 1} g(-\ln (g)) \mathrm{d} y \leq \int_{\Omega} e^{-\nu U}(\nu U+1) \mathrm{d} y+\nu \int_{\Omega} U g \mathrm{~d} y .
$$

Proof of Proposition 2. Integration of (3.2) yields

$$
\mathcal{F}_{\mathrm{P}}(f(t))+\mathcal{F}_{\mathrm{F}}(n(t), u(t))+\int_{0}^{t} \int_{\Omega} \int_{\mathbb{R}^{3}} \frac{D^{2}}{\varepsilon} \mathrm{d} \xi \mathrm{d} x \mathrm{~d} s \leq C_{0},
$$

where $C_{0}$ depends on the initial data. In order to obtain all the conclusions of the claim it suffices to control the negative terms in the free energy functional by the positive terms and prove that all positive terms are uniformly bounded.

By hypotheses (HC3) and (HC8) we can replace $\Phi$ (resp. $\alpha \beta \eta \Phi)$ by $\Phi+C_{1}$ (resp. $\alpha \beta \eta \Phi+C_{2}$ ) and thus, we can assume that the contribution of the potential energies is non negative.

To control the negative term coming from the particles free energy functional, we apply Lemma 1 using (HC9) with $y=(x, v), g(y)=f(x, v)$ and $U(y)=\xi^{2} / 2+\Phi(x)$. We obtain

$$
\begin{aligned}
& \int_{\Omega} \int_{\mathbb{R}^{3}} f \ln ^{+}(f) \mathrm{d} \xi \mathrm{d} x+(1-\nu) \int_{\Omega} \int_{\mathbb{R}^{3}} f\left(\xi^{2} / 2+\Phi\right) \mathrm{d} \xi \mathrm{d} x+\mathcal{F}_{\mathrm{F}}(n(t), u(t)) \\
& \leq C_{0}+\int_{\Omega} \int_{\mathbb{R}^{3}}\left(\nu \xi^{2} / 2+\nu \Phi(x)+1\right) e^{-\nu\left(\xi^{2} / 2+\Phi(x)\right)} \mathrm{d} \xi \mathrm{d} x .
\end{aligned}
$$


Now, we need to control from below the negative term coming from the fluid free energy functional, i.e.,

$$
-\int_{\Omega} \Pi^{-}(n) \mathrm{d} x
$$

This control is quite similar to what it has already been done for the particles free energy. Let us distinguish three cases:

- If $h\left(0^{+}\right)>-\infty$, then $\Pi(n)=\widetilde{\Pi}(n)-h\left(0^{+}\right) n$ with $\widetilde{\Pi}(n) \geq 0$ and thus,

$$
\int_{\Omega} \Pi^{-}(n) \mathrm{d} x \leq h\left(0^{+}\right) \mathcal{M}_{\mathrm{F}}
$$

- If $p(n)=n$, one can use exactly the same argument as for the particles. In fact, Lemma 1 using (HC9) with $y=x, g(y)=n(x)$ and $U(y)=\Phi(x)$ gives

$$
\begin{aligned}
& \int_{\Omega} n \ln ^{+}(n) \mathrm{d} x+(1-\nu) \int_{\Omega} n \Phi \mathrm{d} x+\int_{\Omega} n \frac{|u|^{2}}{2} \mathrm{~d} x \\
& \leq \mathcal{F}_{\mathrm{F}}(n(t), u(t))+\int_{\Omega}(\nu \Phi(x)+1) e^{-\nu \Phi(x)} \mathrm{d} x .
\end{aligned}
$$

- In the general case when $h\left(0^{+}\right)=-\infty$, one proceeds in a similar way to the above arguments using assumptions (HC7) and (HC10). These arguments correspond exactly to [14, Theorem 18, Step 7] and we refer to it for details. As a conclusion, assumptions (HC7) and (HC10) gives us again an estimate of the type

$$
\int_{\Omega} \Pi^{-}(n) \mathrm{d} x \leq B+\nu \int_{\Omega} n \Phi \mathrm{d} x
$$

with $B \in \mathbb{R}$.

As a consequence of the estimates in each case, one is always able to prove that

$$
\int_{\Omega} \Pi^{+}(n) \mathrm{d} x+(1-\nu) \int_{\Omega} n \Phi \mathrm{d} x+\int_{\Omega} n \frac{|u|^{2}}{2} \mathrm{~d} x \leq \mathcal{F}_{\mathrm{F}}(n(t), u(t))+C_{1}
$$

with $C_{1} \in \mathbb{R}$.

Putting together the estimates for the particle and the fluid free energies, one deduces that the positive integrals

$$
\begin{aligned}
& \int_{\Omega} \int_{\mathbb{R}^{3}} f \ln ^{+}(f) \mathrm{d} \xi \mathrm{d} x, \quad \int_{\Omega} \int_{\mathbb{R}^{3}} f\left(\xi^{2} / 2+\Phi\right) \mathrm{d} \xi \mathrm{d} x, \quad \int_{0}^{t} \int_{\Omega} \int_{\mathbb{R}^{3}} \frac{D^{2}}{\varepsilon} \mathrm{d} \xi \mathrm{d} x \mathrm{~d} s \\
& \int_{\Omega} n \frac{|u|^{2}}{2} \mathrm{~d} x, \quad \int_{\Omega} \Pi^{+}(n) \mathrm{d} x \quad \text { and } \quad \int_{\Omega} n \Phi \mathrm{d} x
\end{aligned}
$$


are uniformly bounded. Coming back to the estimate given by Lemma 1 yields the bound on $f \ln ^{-}(f)$. Analogously for the estimate on $\Pi^{-}(n)$ coming back to the estimate listed in each of the above cases.

Let us turn to macroscopic quantities.

Corollary 1. We have the following expansions

$$
J=\rho u+\beta \sqrt{\varepsilon} K, \quad \mathbb{P}=\rho \mathbb{I}+\frac{1}{\beta^{2}} J \otimes u+\sqrt{\varepsilon} \mathbb{K}
$$

where the components of the vector $K$ and of the matrix $\mathbb{K}$ are bounded in $\mathrm{L}^{2}\left(\mathbb{R}^{+} ; \mathrm{L}^{1}(\Omega)\right)$.

Proof. We write $J$ and $\mathbb{P}$ by introducing the entropy dissipation as follows

$$
J=\int_{\mathbb{R}^{3}} u f \mathrm{~d} \xi+\beta \sqrt{\varepsilon} \int_{\mathbb{R}^{3}} \frac{D}{\sqrt{\varepsilon}} \sqrt{f} \mathrm{~d} \xi,
$$

and

$\mathbb{P}=\sqrt{\varepsilon} \int_{\mathbb{R}^{3}} \xi \sqrt{f} \otimes \frac{D}{\sqrt{\varepsilon}} \mathrm{d} \xi+\frac{1}{\beta^{2}} \int_{\mathbb{R}^{3}} \beta \xi \sqrt{f} \otimes u \sqrt{f} \mathrm{~d} \xi-\int_{\mathbb{R}^{3}} \xi \sqrt{f} \otimes 2 \nabla_{\xi} \sqrt{f} \mathrm{~d} \xi$.

Then, the conclusion follows from an application of the Cauchy-Schwarz inequality, and remarking that $\int_{\mathbb{R}^{3}} \xi \sqrt{f} \otimes 2 \nabla_{\xi} \sqrt{f} \mathrm{~d} \xi=-\int_{\mathbb{R}^{3}} f I I \mathrm{~d} \xi$, by integrating by parts.

In what follows, we distinguish two different scalings.

4.2. Flowing Regime. We suppose

$$
\frac{\rho_{\mathrm{P}}}{\rho_{\mathrm{F}}}=\frac{1}{\beta^{2}}, \quad \eta=\beta \quad \text { a fixed positive constant, }
$$

(i.e., $\beta$ does not depend on the small parameter $\varepsilon$; actually this can be slightly relaxed, assuming that $\beta$ tends to some $0<\beta_{\infty}<\infty$ ). Coming back to the physical quantities, this scaling assumption means

$$
\mathcal{V}_{S} \ll \mathrm{U}=\mathcal{V}_{t h},
$$

while $\rho_{\mathrm{P}}$ and $\rho_{\mathrm{F}}$ have the same order. We suppose that $\alpha$ is also a fixed quantity. Note that the ratio $\rho_{\mathrm{P}} / \rho_{\mathrm{F}}$ as well as $\alpha$ can take any fixed value; we only suppose that they remain large compared to $\varepsilon \rightarrow 0$. Therefore, we are concerned with the behavior as $\varepsilon \rightarrow 0$ of

$$
\left\{\begin{array}{l}
\partial_{t} f_{\varepsilon}+\beta\left(\xi \cdot \nabla_{x} f_{\varepsilon}-\nabla_{x} \Phi \cdot \nabla_{\xi} f_{\varepsilon}\right)=\frac{1}{\varepsilon} \operatorname{div}_{\xi}\left(\left(\xi-\frac{1}{\beta} u_{\varepsilon}\right) f+\nabla_{\xi} f_{\varepsilon}\right), \\
\partial_{t}\left(n_{\varepsilon}\right)+\operatorname{div}_{x}\left(n_{\varepsilon} u_{\varepsilon}\right)=0, \\
\partial_{t}\left(n_{\varepsilon} u_{\varepsilon}\right)+\operatorname{Div}_{x}\left(n_{\varepsilon} u_{\varepsilon} \otimes u_{\varepsilon}\right)+\chi \nabla_{x} p\left(n_{\varepsilon}\right)+\alpha \beta^{2} n_{\varepsilon} \nabla_{x} \Phi=\frac{1}{\varepsilon \beta^{2}}\left(J_{\varepsilon}-\rho_{\varepsilon} u_{\varepsilon}\right),
\end{array}\right.
$$


with

$$
\left(\begin{array}{c}
\rho_{\varepsilon} \\
J_{\varepsilon} \\
\mathbb{P}_{\varepsilon}
\end{array}\right)(t, x)=\int_{\mathbb{R}^{3}}\left(\begin{array}{l}
1 \\
\beta \xi \\
\xi \otimes \xi
\end{array}\right) f_{\varepsilon}(t, x, x i) \mathrm{d} \xi
$$

The macroscopic quantities satisfy the following moment system

$$
\left\{\begin{array}{l}
\partial_{t} \rho_{\varepsilon}+\operatorname{div}_{x} J_{\varepsilon}=0, \\
\frac{1}{\beta^{2}} \partial_{t} J_{\varepsilon}+\operatorname{Div}_{x} \mathbb{P}_{\varepsilon}+\rho_{\varepsilon} \nabla_{x} \Phi=-\frac{1}{\varepsilon \beta^{2}}\left(J_{\varepsilon}-\rho_{\varepsilon} u_{\varepsilon}\right) .
\end{array}\right.
$$

Using the fluid momentum equation, we are led to

$\partial_{t}\left(n_{\varepsilon} u_{\varepsilon}+\beta^{-2} J_{\varepsilon}\right)+\operatorname{Div}_{x}\left(n_{\varepsilon} u_{\varepsilon} \otimes u_{\varepsilon}+\mathbb{P}_{\varepsilon}\right)+\chi \nabla_{x} p\left(n_{\varepsilon}\right)+\left(\alpha \beta^{2} n_{\varepsilon}+\rho_{\varepsilon}\right) \nabla_{x} \Phi=0$.

Now, coming back to (4.1), it is tempting to infer

$$
J_{\varepsilon} \simeq \rho_{\varepsilon} u_{\varepsilon}, \quad \mathbb{P}_{\varepsilon} \simeq \rho_{\varepsilon} I I+\beta^{-2} \rho_{\varepsilon} u_{\varepsilon} \otimes u_{\varepsilon},
$$

which is made more precise in Corollary 1.

Theorem 3. Let $f_{\varepsilon}, n_{\varepsilon}, u_{\varepsilon}$ be a family of solutions of (4.3). Let us suppose that, as $\varepsilon \rightarrow 0$,

$$
\rho_{\varepsilon} \rightarrow \rho, \quad n_{\varepsilon} \rightarrow n, \quad u_{\varepsilon} \rightarrow u
$$

in the sense of distributions. Suppose also that the non linear terms pass to the limit

$\rho_{\varepsilon} u_{\varepsilon} \rightarrow \rho u, \quad \rho_{\varepsilon} u_{\varepsilon} \otimes u_{\varepsilon} \rightarrow \rho u \otimes u, \quad n_{\varepsilon} u_{\varepsilon} \otimes u_{\varepsilon} \rightarrow n u \otimes u, \quad p\left(n_{\varepsilon}\right) \rightarrow p(n)$

in $\mathcal{D}^{\prime}\left(\mathbb{R}^{+} \times \Omega\right)$. Then, $(\rho, n, u)$ satisfy the following system

$$
\left\{\begin{aligned}
\partial_{t} \rho+\operatorname{div}_{x}(\rho u)= & \\
\partial_{t} n+\operatorname{div}_{x}(n u)= & 0 \\
\partial_{t}\left(\left(n+\beta^{-2} \rho\right) u\right) & +\operatorname{Div}_{x}\left(\left(n+\beta^{-2} \rho\right) u \otimes u\right) \\
& +\nabla_{x}(\rho+\chi p(n))+\left(\alpha \beta^{2} n+\rho\right) \nabla_{x} \Phi=0 .
\end{aligned}\right.
$$

4.3. Bubbling Regime. In this case, we assume

$$
\frac{\rho_{\mathrm{P}}}{\rho_{\mathrm{F}}}=\frac{1}{\beta^{2}}, \quad \eta=\beta, \quad \beta=\frac{1}{\sqrt{\varepsilon}},
$$

and thus, according to (3.1), we also have

$$
\frac{\rho_{\mathrm{P}}}{\rho_{\mathrm{F}}}=\varepsilon, \quad \eta=\frac{1}{\sqrt{\varepsilon}} .
$$


(This can be slightly modified to $\beta-\beta_{\infty} / \sqrt{\varepsilon} \rightarrow 0$ with $0<\beta_{\infty}<\infty$.) Coming back to the physical quantities, it means that

$$
\mathcal{V}_{S}=\mathrm{U} \ll \mathcal{V}_{t h}
$$

Finally, we assume that $\alpha=\operatorname{sign}(\alpha) \varepsilon$ with $\operatorname{sign}(\alpha)= \pm 1$. Therefore, we are concerned with the behavior as $\varepsilon \rightarrow 0$ of

$$
\left\{\begin{array}{l}
\partial_{t} f_{\varepsilon}+\frac{1}{\sqrt{\varepsilon}}\left(\xi \cdot \nabla_{x} f_{\varepsilon}-\nabla_{x} \Phi \cdot \nabla_{\xi} f_{\varepsilon}\right)=\frac{1}{\varepsilon} \operatorname{div}_{\xi}\left(\left(\xi-\sqrt{\varepsilon} u_{\varepsilon}\right) f+\nabla_{\xi} f_{\varepsilon}\right), \\
\partial_{t} n_{\varepsilon}+\operatorname{div}_{x}\left(n_{\varepsilon} u_{\varepsilon}\right)=0, \\
\partial_{t}\left(n_{\varepsilon} u_{\varepsilon}\right)+\operatorname{Div}_{x}\left(n_{\varepsilon} u_{\varepsilon} \otimes u_{\varepsilon}\right)+\chi \nabla_{x} p\left(n_{\varepsilon}\right)+\operatorname{sign}(\alpha) n_{\varepsilon} \nabla_{x} \Phi=\left(J_{\varepsilon}-\rho_{\varepsilon} u_{\varepsilon}\right),
\end{array}\right.
$$

with

$$
\rho_{\varepsilon}(t, x)=\int_{\mathbb{R}^{3}} f_{\varepsilon}(t, x, \xi) \mathrm{d} \xi, \quad J_{\varepsilon}(t, x)=\int_{\mathbb{R}^{3}} \frac{1}{\sqrt{\varepsilon}} \xi f_{\varepsilon}(t, x, \xi) \mathrm{d} \xi .
$$

We still denote

$$
\mathbb{P}_{\varepsilon}(t, x)=\int_{\mathbb{R}^{3}} \xi \otimes \xi f_{\varepsilon}(t, x, \xi) \mathrm{d} \xi .
$$

Then, we obtain the following moment equations

$$
\left\{\begin{array}{l}
\partial_{t} \rho_{\varepsilon}+\operatorname{div}_{x} J_{\varepsilon}=0, \\
\varepsilon \partial_{t} J_{\varepsilon}+\operatorname{Div}_{x} \mathbb{P}_{\varepsilon}+\rho_{\varepsilon} \nabla_{x} \Phi=-\left(J_{\varepsilon}-\rho_{\varepsilon} u_{\varepsilon}\right) .
\end{array}\right.
$$

Using the formal ansatz (4.1), and Corollary 1, the distribution function can be approximated as $f_{\varepsilon} \simeq \frac{\rho_{\varepsilon}}{(2 \pi)^{3 / 2}} e^{-\xi^{2} / 2}$, and $\mathbb{P}_{\varepsilon} \simeq \rho_{\varepsilon} I$ I , but it remains to describe the behavior of $J_{\varepsilon}$. Letting $\varepsilon$ go to 0 in the first order moment equation yields

$$
\nabla_{x} \rho+\rho \nabla_{x} \Phi=-J+\rho u .
$$

Inserting this result in the continuity equation, and passing to the limit in the fluid equation, we are led to the following claim.

Theorem 4. Let $f_{\varepsilon}, n_{\varepsilon}, u_{\varepsilon}$ be a family of solutions of (4.7). Let us suppose that

$$
\rho_{\varepsilon} \rightarrow \rho, \quad J_{\varepsilon} \rightarrow J, \quad n_{\varepsilon} \rightarrow n, \quad u_{\varepsilon} \rightarrow u
$$

in the sense of distributions. Suppose also that the non linear terms pass to the limit

$\rho_{\varepsilon} u_{\varepsilon} \rightarrow \rho u, \quad \sqrt{\varepsilon} J_{\varepsilon} \otimes u_{\varepsilon} \rightarrow 0, \quad n_{\varepsilon} u_{\varepsilon} \otimes u_{\varepsilon} \rightarrow n u \otimes u, \quad p\left(n_{\varepsilon}\right) \rightarrow p(n)$ 
in $\mathcal{D}^{\prime}\left(\mathbb{R}^{+} \times \Omega\right)$. Then, $J=\rho\left(u-\nabla_{x} \Phi\right)-\nabla_{x} \rho$, and $(\rho, n, u)$ satisfy the following system

(4.9)

$$
\left\{\begin{array}{l}
\partial_{t} \rho+\operatorname{div}_{x}\left(\rho\left(u-\nabla_{x} \Phi\right)-\nabla_{x} \rho\right)=0, \\
\partial_{t} n+\operatorname{div}_{x}(n u)=0, \\
\partial_{t}(n u)+\operatorname{Div}_{x}(n u \otimes u)+\nabla_{x}(\chi p(n)+\rho)+(\operatorname{sign}(\alpha) n+\rho) \nabla_{x} \Phi=0 .
\end{array}\right.
$$

Remark 7. The continuity equation for the particle density $\rho$ in (4.9) is often referred as the Smoluchowski equation. Note that in the limit equations (4.9), the behavior of the fluid remains dependent on the evolution of the particles, even in absence of external forces, through the $\nabla_{x} \rho$. It is worth pointing out the difference with incompressible models, as studied in [28], where this term disappears due to the incompressibility constraint.

Remark 8. For both the flowing and the bubbling regimes, the scaling assumption on the parameter $\alpha$, which measures the ratio of the effect of the external force on the fluid and on the particles, is particularly clear when looking at the example of gravity force. Indeed, we have seen that $\alpha$ is a simple function of $\rho_{\mathrm{P}} / \rho_{\mathrm{F}}$ which then imposes the scaling behavior.

Remark 9. For both regimes, the available bounds, provided by the entropy dissipation, leads to some weak compactness properties on the sequences $f_{\varepsilon}$, $\rho_{\varepsilon}, n_{\varepsilon}$ and $\sqrt{n_{\varepsilon}} u_{\varepsilon}$. However, we do not have any estimate on the product $\rho_{\varepsilon} u_{\varepsilon}$, and thus on $J_{\varepsilon}$. Therefore, this is certainly a great mathematical difficulty when dealing with a rigorous proof of convergence. When NavierStokes equation is considered, one obtains an additional $\mathrm{H}^{1}$ estimate. Then, in dimension two, this combines with the $\mathrm{L} \ln \mathrm{L}$ estimate on $\rho$, so that $\rho_{\varepsilon} u_{\varepsilon}$ is a bounded sequence of integrable functions which passes to the limit, see [28]. We refer to [40] for an attempt dealing with a relative entropy approach.

Remark 10. When considering the problem in a bounded domain, boundary conditions have also to be discussed for the limit equations. For the flowing regime the physical boundary condition $u \cdot \nu(x)=0$ on $\partial \Omega$, preserved by the asymptotic procedure, is enough since $\rho$ and $n$ satisfy the same transport equation with velocity $u$. For the bubbling regime, the limit equation for the particles density is a convection-diffusion equation. While the boundary condition for the equation in $n$ is still the physical boundary condition $u$. $\nu(x)=0$ on $\partial \Omega$, the boundary condition for the equation in $\rho$, which comes from the asymptotic analysis, is the no-flux boundary condition

$$
\rho\left(\nabla_{x} \Phi-u\right) \cdot \nu(x)+\partial_{\nu} \rho=\rho \nabla_{x} \Phi \cdot \nu(x)+\partial_{\nu} \rho=0
$$


on $\partial \Omega$. We refer to $[39,7]$ for similar considerations and rigorous proofs in a different physical context.

\section{Properties of the Limit Systems}

We will show that both limit systems (4.6) and (4.9) have first order terms with a hyperbolic structure while keeping the main qualitative property of the original Vlasov-Euler system, that is, the nonlinear stability of steady states.

5.1. Hyperbolicity. Consider the equivalent flowing regime system to (4.6) given by

$$
\left\{\begin{array}{l}
\partial_{t} \rho+\operatorname{div}_{x}(\rho u)=0 \\
\partial_{t} r+\operatorname{div}_{x}(r u)=0 \\
\partial_{t}(r u)+\operatorname{Div}_{x}(r u \otimes u)+\nabla_{x}(\rho+\chi p(n))+\alpha \beta^{2} r \nabla_{x} \Phi=0,
\end{array}\right.
$$

with $r=n+\beta^{-2} \rho$. Therefore, the first order term has a flux function $F_{f r}: \mathbb{R}^{5} \longrightarrow M_{5 \times 3}(\mathbb{R})$ given by:

$$
F_{f r}(U)=\left(\frac{j \rho}{r}, j, \frac{j \otimes j}{r}+\left(\chi p\left(r-\beta^{-2} \rho\right)+\rho\right) I I\right)
$$

with $U=(\rho, r, j)$ and $j=r u$. Taking the component in the $x_{1}$-direction given by

$$
F_{f r}^{1}(U)=\left(\frac{j_{1} \rho}{r}, j_{1}, \frac{j_{1}^{2}}{r}+\chi p\left(r-\beta^{-2} \rho\right)+\rho, \frac{j_{1} j_{2}}{r}, \frac{j_{1} j_{3}}{r}\right),
$$

it is easy to check that its jacobian matrix has real eigenvalues given by $\frac{j_{1}}{r}$ (triple) and two simple eigenvalues $\frac{j_{1}}{r} \pm \sqrt{\frac{\rho}{r}+\chi p^{\prime}(n) \frac{n}{r}}$. Therefore, the system (5.1) is hyperbolic.

Regarding the bubbling regime system (4.9), we see that this system consists in a drift-diffusion equation for the particle density $\rho$ coupled with a system on $(n, j=n u)$ which is clearly hyperbolic. Alternatively, one can write an equivalent system to (4.9) by the standard change of variable $r=\rho e^{\Phi}$ obtaining:

$$
\left\{\begin{array}{l}
\partial_{t} r+\operatorname{div}_{x}(r u)=r\left(u \cdot \nabla_{x} \Phi\right)+e^{\Phi} \operatorname{div}_{x}\left(e^{-\Phi} \nabla_{x} r\right), \\
\partial_{t} n+\operatorname{div}_{x}(n u)=0, \\
\partial_{t}(n u)+\operatorname{Div}_{x}(n u \otimes u)+\nabla_{x}\left(\chi p(n)+r e^{-\Phi}\right)+\left(\operatorname{sign}(\alpha) n+r e^{-\Phi}\right) \nabla_{x} \Phi=0,
\end{array}\right.
$$

for which the first order term gives a hyperbolic system. 
5.2. Nonlinear Stability. Both limit systems: the flowing and the bubbling regime systems keep the remarkable property of the original VlasovEuler system. Both systems have a natural entropy which implies the nonlinear stability of the corresponding steady states.

Following the ideas of Section 3, it is easy to guess the following free energy functional:

$(5.2) \mathcal{F}_{f r}(\rho, n, u)=\int_{\Omega}\left[\left(n+\beta^{-2} \rho\right) \frac{|u|^{2}}{2}+\chi \Pi(n)+\rho \ln \rho+\left(\alpha \beta^{2} n+\rho\right) \Phi\right] \mathrm{d} x$, for the flowing regime system (4.6). It can be checked easily that:

$$
\frac{\mathrm{d}}{\mathrm{d} t} \mathcal{F}_{f r}(\rho(t), n(t), u(t))=0
$$

since we have the following identities:

- Entropy of the particles

$$
\frac{\mathrm{d}}{\mathrm{dt}} \int_{\Omega} \rho \ln \rho \mathrm{d} x=\int_{\Omega} u \cdot \nabla_{x} \rho \mathrm{d} x
$$

- Entropy of the fluid

$$
\frac{\mathrm{d}}{\mathrm{dt}} \int_{\Omega} \Pi(n) \mathrm{d} x=\int_{\Omega} u \cdot \nabla_{x} p(n) \mathrm{d} x .
$$

- Potential energy of the particles and fluid

$$
\frac{\mathrm{d}}{\mathrm{dt}} \int_{\Omega}\left(\alpha \beta^{2} n+\rho\right) \Phi \mathrm{d} x=\int_{\Omega}\left(\alpha \beta^{2} n+\rho\right) u \cdot \nabla_{x} \Phi \mathrm{d} x .
$$

- Kinetic energy of the particles and fluid

$$
\begin{aligned}
\frac{\mathrm{d}}{\mathrm{dt}} \int_{\Omega}\left(n+\beta^{-2} \rho\right) \frac{|u|^{2}}{2} \mathrm{~d} x= & -\int_{\Omega} u \cdot \nabla_{x}(\chi p(n)+\rho) \mathrm{d} x \\
& -\int_{\Omega}\left(\alpha \beta^{2} n+\rho\right) u \cdot \nabla_{x} \Phi \mathrm{d} x .
\end{aligned}
$$

Therefore, analogously to Section 3, one considers the free energy functional $\mathcal{F}_{f r}(\rho, n, u)$ that has a unique minimizer in $\mathrm{L}_{+}^{1}(\Omega) \times \mathrm{L}_{+}^{1}(\Omega) \times \mathrm{L}^{2}\left(\Omega, \mathbb{R}^{3}\right)$ with total particles and fluid masses equal to $\mathcal{M}_{\mathrm{P}}$ and $\mathcal{M}_{\mathrm{F}}$ respectively. This minimizer by Theorem 1 is given by $\left(\rho_{S}, n_{S}, u_{S}=0\right)$ where $\rho_{S}$ is given by

$$
\rho_{S}(x)=\int_{\mathbb{R}^{3}} f_{S} \mathrm{~d} \xi=\mathcal{M}_{\mathrm{P}} \frac{e^{-\Phi(x)}}{\int_{\Omega} e^{-\Phi(x)} \mathrm{d} x}=Z_{\mathcal{M}_{\mathrm{P}}}^{\rho} e^{-\Phi(x)}
$$

and $n_{S}$ given by (3.6) where $\eta=\beta$. We can define the relative entropy in the flowing regime case as

$R E_{f r}\left((\rho, n, u) \mid\left(\rho_{S}, n_{S}, u_{S}\right)\right)=\mathcal{F}_{f r}(\rho, n, u)-\ln \left(Z_{\mathcal{M}_{\mathrm{P}}}^{\rho}\right) \mathcal{M}_{\mathrm{P}}-\chi E_{\mathrm{F}}\left(n_{S}\right) \geq 0$, 
and again, this relative entropy functional

$$
\begin{aligned}
R E_{f r}\left((\rho, n, u) \mid\left(\rho_{S}, n_{S}, u_{S}\right)\right) & =\int_{\Omega}\left(\rho \ln \left(\frac{\rho}{\rho_{S}}\right)-\rho+\rho_{S}\right) \mathrm{d} x \\
& +\int_{\Omega} n \frac{|u|^{2}}{2} \mathrm{~d} x+\chi\left(E_{\mathrm{F}}(n)-E_{\mathrm{F}}\left(n_{S}\right)\right)
\end{aligned}
$$

vanishes if and only if $\rho=\rho_{S}, n=n_{S}$ and $u=u_{S}=0$. Moreover, we easily obtain the following non-linear stability result.

Theorem 5. Given $(\rho(t), n(t), u(t))$ a solution to the Cauchy problem for the system (4.6) and $\left(\rho_{S}, n_{S}, u_{S}=0\right)$ as above, such that

$$
\int_{\Omega} \rho_{0} \mathrm{~d} x=\int_{\Omega} \rho_{S} \mathrm{~d} x \quad \text { and } \quad \int_{\Omega} n_{0} \mathrm{~d} x=\int_{\Omega} n_{S} \mathrm{~d} x,
$$

then for any $\epsilon>0$, there exists $\delta>0$ such that if

$$
R E_{f r}\left(\left(\rho_{0}, n_{0}, u_{0}\right) \mid\left(\rho_{S}, n_{S}, u_{S}\right)\right) \leq \delta,
$$

we conclude the solution satisfies

$$
\left\|\rho(t)-\rho_{S}\right\|_{\mathrm{L}^{1}\left(\Omega \times \mathbb{R}^{3}\right)} \leq \epsilon,\left\|n(t)-n_{S}\right\|_{\mathrm{L}^{1}(\Omega)} \leq \epsilon \text { and } \int_{\Omega} n(t) \frac{|u(t)|^{2}}{2} \mathrm{~d} x \leq \epsilon
$$

for all $t \geq 0$.

For the bubbling regime system (4.9), we can proceed analogously, and find the following free energy functional

$$
\mathcal{F}_{b r}(\rho, n, u)=\int_{\Omega}\left[n \frac{|u|^{2}}{2}+\chi \Pi(n)+\rho \ln \rho+(\operatorname{sign}(\alpha) n+\rho) \Phi\right] \mathrm{d} x,
$$

which in this case is dissipated along the flow, i.e.,

$$
\frac{\mathrm{d}}{\mathrm{d} t} \mathcal{F}_{b r}(\rho(t), n(t), u(t))=-\int_{\Omega} \rho\left|\nabla_{x} \Phi+\nabla_{x} \ln \rho\right|^{2} \mathrm{~d} x \leq 0 .
$$

A completely analogous non-linear stability result to Theorem 5 can be written in the bubbling regime case, being the stationary state $\left(\rho_{S}, n_{S}, u_{S}=\right.$ 0 ) with $\rho_{S}$ given by (5.4) and $n_{S}$ given by (3.6) where the constant $\alpha \beta \eta$ is substituted by $\operatorname{sign}(\alpha)$.

\section{Asymptotic Analysis of the Linearized Problem}

It is certainly difficult to rigorously justify the asymptotics described in Section 4. However, we can validate our approach by the analysis of the linearized version of the problem. To this end, let us go back to (2.2), and expand the solution as follows

$$
\left\{\begin{array}{l}
f(t, x, \xi)=M(\xi)+\sigma_{\mathrm{P}} \widetilde{f}(t, x, \xi), \\
n(t, x)=\bar{n}+\sigma_{\mathrm{F}} \widetilde{n}(t, x), \quad u(t, x)=\sigma_{\mathrm{F}} \widetilde{u}(t, x),
\end{array}\right.
$$


where $M(\xi)=(2 \pi)^{-3 / 2} e^{-\xi^{2} / 2}$ and $\bar{n}$ is a given positive constant. We shall discuss the choice of the parameters $\sigma_{\mathrm{P}, \mathrm{F}}$ by means of the other dimensionless coefficients. Inserting the ansatz in (2.2) and dropping all terms with high power of the $\sigma_{\mathrm{P}, \mathrm{F}}$ 's, we get (6.1)

$$
\left\{\begin{array}{l}
\partial_{t} \tilde{f}+\beta \xi \cdot \nabla_{x} \tilde{f}-\eta \nabla_{x} \Phi \cdot \nabla_{\xi} \tilde{f} \\
\quad=\frac{1}{\varepsilon} \operatorname{div}_{\xi}\left(\xi \tilde{f}+\nabla_{\xi} \widetilde{f}\right)+\left(\frac{\eta}{\sigma_{\mathrm{P}}} \nabla_{x} \Phi-\frac{\sigma_{\mathrm{F}}}{\varepsilon \beta \sigma_{\mathrm{P}}} \widetilde{u}\right) \cdot \nabla_{\xi} M, \\
\partial_{t} \widetilde{n}+\bar{n} \operatorname{div}_{x} \widetilde{u}=0, \\
\bar{n} \partial_{t} \widetilde{u}+p^{\prime}(\bar{n}) \nabla_{x} \widetilde{n}+\alpha \frac{\beta \eta}{\sigma_{\mathrm{F}}} \bar{n} \nabla_{x} \Phi=\frac{1}{\varepsilon} \frac{\rho_{\mathrm{P}}}{\rho_{\mathrm{F}}}\left(\frac{\sigma_{\mathrm{P}}}{\sigma_{\mathrm{F}}} \widetilde{J}-\widetilde{u}\right),
\end{array}\right.
$$

where

$$
\widetilde{\rho}(t, x)=\int_{\mathbb{R}^{3}} \tilde{f}(t, x, \xi) \mathrm{d} \xi, \quad \widetilde{J}(t, x)=\beta \int_{\mathbb{R}^{3}} \xi \widetilde{f}(t, x, \xi) \mathrm{d} \xi
$$

We expect that the Fokker-Planck operator is the leading term in the equation, and that both force terms associated with the Maxwellian have the same order, a step higher than $\nabla_{x} \Phi \cdot \nabla_{\xi} \tilde{f}$ :

$$
\frac{\eta}{\sigma_{\mathrm{P}}}=\frac{\sigma_{\mathrm{F}}}{\varepsilon \beta \sigma_{\mathrm{P}}}=\frac{1}{\sqrt{\varepsilon}}, \quad \eta=1
$$

Furthermore, the moment equations now read

$$
\begin{aligned}
& \partial_{t} \widetilde{\rho}+\operatorname{div}_{x} \widetilde{J}=0 \\
& \partial_{t} \widetilde{J}+\beta^{2} \operatorname{Div}_{x}\left(\int \xi \otimes \xi \widetilde{f} \mathrm{~d} \xi\right)+\beta \eta \widetilde{\rho} \nabla_{x} \Phi=-\frac{1}{\varepsilon} \widetilde{J}+\frac{\beta}{\sqrt{\varepsilon}}\left(\widetilde{u}-\nabla_{x} \Phi\right) .
\end{aligned}
$$

We require that the terms in the right hand side contribute at the same order, which gives $\beta=1 / \sqrt{\varepsilon}$. In turn, we get $\sigma_{\mathrm{P}}=\sigma_{\mathrm{F}}=\sqrt{\varepsilon}$. Eventually, we assume that all force terms in the fluid momentum equation are of order $\mathcal{O}(1)$; which motivates $\rho_{\mathrm{P}} / \rho_{\mathrm{F}}=\varepsilon$, and $\alpha=\operatorname{sign}(\alpha) \varepsilon$. This still agrees with the formula of $\alpha$ for the gravity force.

Coming back to the physical interpretation, we linearize around a constant state, with zero bulk velocity, at order $\sqrt{\varepsilon}$ and we assume

$$
\mathcal{V}_{S} \ll \mathrm{U} \ll \mathcal{V}_{t h}, \quad \rho_{\mathrm{P}} \ll \rho_{\mathrm{F}}, \quad \alpha \ll 1
$$


Summarizing, we wish to investigate the asymptotic behavior $\varepsilon \rightarrow 0$ of the following problem

(6.2)

$$
\left\{\begin{array}{l}
\partial_{t} f_{\varepsilon}+\frac{1}{\sqrt{\varepsilon}} \xi \cdot \nabla_{x} f_{\varepsilon}-\nabla_{x} \Phi \cdot \nabla_{\xi} f_{\varepsilon} \\
\quad=\frac{1}{\varepsilon} \operatorname{div}_{\xi}\left(\xi f_{\varepsilon}+\nabla_{\xi} f_{\varepsilon}\right)+\frac{1}{\sqrt{\varepsilon}}\left(u_{\varepsilon}-\nabla_{x} \Phi\right) \cdot \xi M(\xi), \\
\partial_{t} n_{\varepsilon}+\bar{n} \operatorname{div}_{x} u_{\varepsilon}=0, \\
\bar{n} \partial_{t} u_{\varepsilon}+p^{\prime}(\bar{n}) \nabla_{x} n_{\varepsilon}+\operatorname{sign}(\alpha) \bar{n} \nabla_{x} \Phi=\frac{1}{\sqrt{\varepsilon}} \int_{\mathbb{R}^{3}} \xi f_{\varepsilon} \mathrm{d} \xi-u_{\varepsilon}=J_{\varepsilon}-u_{\varepsilon},
\end{array}\right.
$$

with $J_{\varepsilon}(t, x)=\frac{1}{\sqrt{\varepsilon}} \int_{\mathbb{R}^{3}} \xi f_{\varepsilon} \mathrm{d} \xi$.

Some straightforward manipulations leads to the following entropy relation

$$
\begin{aligned}
\frac{\mathrm{d}}{\mathrm{dt}}\left(\frac{1}{2} \int_{\mathbb{R}^{3}} \int_{\mathbb{R}^{3}} \frac{\left|f_{\varepsilon}\right|^{2}}{M(\xi)} \mathrm{d} \xi \mathrm{d} x+\int_{\mathbb{R}^{3}} \int_{\mathbb{R}^{3}} f_{\varepsilon} \Phi \mathrm{d} \xi \mathrm{d} x\right. \\
\left.\quad+\bar{n} \int_{\mathbb{R}^{3}} \frac{\left|u_{\varepsilon}\right|^{2}}{2} \mathrm{~d} x+p^{\prime}(\bar{n}) \int_{\mathbb{R}^{3}} \frac{\left|n_{\varepsilon}\right|^{2}}{2} \mathrm{~d} x+\operatorname{sign}(\alpha) \int_{\mathbb{R}^{3}} n_{\varepsilon} \Phi \mathrm{d} x\right) \\
\quad+\frac{1}{\varepsilon} \int_{\mathbb{R}^{3}} \int_{\mathbb{R}^{3}} \frac{\left|\xi f_{\varepsilon}-\sqrt{\varepsilon} u_{\varepsilon} M(\xi)+\nabla_{\xi} f_{\varepsilon}\right|^{2}}{M(\xi)} \mathrm{d} \xi \mathrm{d} x \\
=-\int_{\mathbb{R}^{3}} \int_{\mathbb{R}^{3}} \nabla_{x} \Phi f \cdot \nabla_{\xi}\left(\frac{f}{M}\right) \mathrm{d} \xi \mathrm{d} x .
\end{aligned}
$$

Using Cauchy-Schwarz and Young inequalities, the right hand side can be evaluated by

$$
\begin{aligned}
\frac{1}{2 \varepsilon} \int_{\mathbb{R}^{3}} \int_{\mathbb{R}^{3}} \frac{\left|\xi f_{\varepsilon}-\sqrt{\varepsilon} u_{\varepsilon} M(\xi)+\nabla_{\xi} f_{\varepsilon}\right|^{2}}{M(\xi)} \mathrm{d} \xi \mathrm{d} x \\
\quad+\left(\frac{1}{2}+\frac{\varepsilon}{2}\left\|\nabla_{x} \Phi\right\|_{L^{\infty}\left(\mathbb{R}^{3}\right)}^{2}\right) \int_{\mathbb{R}^{3}} \int_{\mathbb{R}^{3}} \frac{\left|f_{\varepsilon}\right|^{2}}{M(\xi)} \mathrm{d} \xi \mathrm{d} x+\frac{1}{2} \int_{\mathbb{R}^{3}}\left|u_{\varepsilon}\right|^{2} \mathrm{~d} x .
\end{aligned}
$$

Using the Gronwall lemma, we deduce the following a priori estimates.

Lemma 2. Let $f_{\varepsilon}, n_{\varepsilon}, u_{\varepsilon}$ be a family of solutions of (6.2) associated to initial data $f_{\varepsilon, 0} \geq 0, n_{\varepsilon, 0} \geq 0, u_{\varepsilon, 0}$ such that

$$
\begin{aligned}
\sup _{\varepsilon>0}\left\{\int_{\mathbb{R}^{3}} \int_{\mathbb{R}^{3}}\left(f_{\varepsilon, 0}+\frac{\left|f_{\varepsilon, 0}\right|^{2}}{M(\xi)}+f_{\varepsilon, 0}|\Phi|\right) \mathrm{d} \xi \mathrm{d} x\right. \\
\left.\quad+\int_{\mathbb{R}^{3}}\left(n_{\varepsilon, 0}+\frac{\left|n_{\varepsilon, 0}\right|^{2}}{2}+\frac{\left|u_{\varepsilon, 0}\right|^{2}}{2}+n_{\varepsilon, 0}|\Phi|\right) \mathrm{d} x\right\}<\infty .
\end{aligned}
$$


We also suppose that $\nabla_{x} \Phi \in \mathrm{L}^{\infty}\left(\mathbb{R}^{3}\right)$ and

$$
\begin{aligned}
& \text { either } \operatorname{sign}(\alpha)=1 \text { and } \Phi(x) \geq C \text { a.e. } x \in \Omega, \\
& \text { or } \operatorname{sign}(\alpha)=-1 \text { and } \Phi \in \mathrm{L}^{\infty}\left(\mathbb{R}^{3}\right) \text {. }
\end{aligned}
$$

Let $0<T<\infty, 0<\varepsilon<1$. Then, the following properties hold:

(i) $\left(f_{\varepsilon}\right)_{\varepsilon>0}$ is bounded in $\mathrm{L}^{\infty}\left(\mathbb{R}^{+} ; \mathrm{L}^{1}\left(\mathbb{R}^{3} \times \mathbb{R}^{3}\right)\right),\left(\Phi f_{\varepsilon}\right)_{\varepsilon>0}$ is bounded in $\mathrm{L}^{\infty}\left(0, T ; \mathrm{L}^{1}\left(\mathbb{R}^{3} \times \mathbb{R}^{3}\right)\right)$, and moreover $\left(f_{\varepsilon} / \sqrt{M(\xi)}\right)_{\varepsilon>0}$ is bounded in $\mathrm{L}^{\infty}\left(0, T ; \mathrm{L}^{2}\left(\mathbb{R}^{3} \times \mathbb{R}^{3}\right)\right)$.

(ii) $\left(\frac{1}{\sqrt{\varepsilon M(\xi)}}\left|\xi f_{\varepsilon}-\sqrt{u}_{\varepsilon} M(\xi)+\nabla_{\xi} f_{\varepsilon}\right|=\frac{1}{\sqrt{\varepsilon}} D_{\varepsilon}\right)_{\varepsilon>0}$ is bounded in $\mathrm{L}^{2}\left((0, T) \times \mathbb{R}^{3} \times \mathbb{R}^{3}\right)$.

(iii) $\left(\rho_{\varepsilon}\right)_{\varepsilon>0}$ is bounded in $\mathrm{L}^{\infty}\left(0, T ; \mathrm{L}^{2}\left(\mathbb{R}^{3}\right)\right) \cap \mathrm{L}^{\infty}\left(\mathbb{R}^{+} ; \mathrm{L}^{1}\left(\mathbb{R}^{3}\right)\right)$,

(iv) $\left(J_{\varepsilon}\right)_{\varepsilon>0}$ is bounded in $\mathrm{L}^{2}\left((0, T) \times \mathbb{R}^{3}\right)$.

(v) $\mathbb{P}_{\varepsilon}=\rho_{\varepsilon} I I+\sqrt{\varepsilon} \mathbb{R}_{\varepsilon}$, with $\left(\mathbb{R}_{\varepsilon}\right)_{\varepsilon>0}$ bounded in $\mathrm{L}^{2}\left((0, T) \times \mathbb{R}^{3}\right)$.

(vi) $\left(n_{\varepsilon}\right)_{\varepsilon>0}$ and $\left(u_{\varepsilon}\right)_{\varepsilon>0}$ are bounded in $\mathrm{L}^{\infty}\left(0, T ; \mathrm{L}^{2}\left(\mathbb{R}^{3}\right)\right)$.

Proof. Obviously, the mass conservation

$$
\frac{\mathrm{d}}{\mathrm{d} t} \int_{\mathbb{R}^{3}} \int_{\mathbb{R}^{3}} f_{\varepsilon} \mathrm{d} \xi \mathrm{d} x=0, \quad \int_{\mathbb{R}^{3}} n_{\varepsilon} \mathrm{d} \xi \mathrm{d} x=0
$$

hold. Then, by using (6.4), the potential energy contribution is bounded from below. Thus, we deduce readily (i), (ii) and (vi) from (6.3). Next, the Cauchy-Schwarz inequality implies the following estimates

$$
\left|\rho_{\varepsilon}(t, x)\right|^{2} \leq \int_{\mathbb{R}^{3}} M \mathrm{~d} \xi \int_{\mathbb{R}^{3}} \frac{\left|f_{\varepsilon}\right|^{2}}{M} \mathrm{~d} \xi,
$$

and

$$
\begin{aligned}
\left|J_{\varepsilon}(t, x)\right|^{2} & =\left|\int_{\mathbb{R}^{3}} \frac{D_{\varepsilon}}{\sqrt{\varepsilon}} \sqrt{M} \mathrm{~d} \xi+u_{\varepsilon} \int_{\mathbb{R}^{3}} M \mathrm{~d} \xi\right|^{2} \\
& \leq 2\left(\int_{\mathbb{R}^{3}} \frac{\left|D_{\varepsilon}\right|^{2}}{\varepsilon} \mathrm{d} \xi+\left|u_{\varepsilon}\right|^{2}\right)
\end{aligned}
$$

which proves (iii) and (iv). Finally, we write

$$
\mathbb{P}_{\varepsilon}(t, x)=\sqrt{\varepsilon} \int_{\mathbb{R}^{3}} \xi \sqrt{M} \otimes \frac{D_{\varepsilon}}{\sqrt{\varepsilon}} \mathrm{d} \xi+\int_{\mathbb{R}^{3}}\left(\sqrt{\varepsilon} \xi \otimes u_{\varepsilon} M-\xi \otimes \nabla_{\xi} f_{\varepsilon}\right) \mathrm{d} \xi .
$$

The last integral is equal to $\rho_{\varepsilon} \mathbb{I}$, by using an integration by parts. An application of the Cauchy-Schwarz inequality proves that the first integral reads $\sqrt{\varepsilon} \mathbb{R}_{\varepsilon}(t, x)$, with $\mathbb{R}_{\varepsilon}$ bounded in $\mathrm{L}^{2}\left((0, T) \times \mathbb{R}^{3}\right)$.

Hence, for any $0<T<\infty$, we can assume that $\rho_{\varepsilon}, J_{\varepsilon}, n_{\varepsilon}$ and $u_{\varepsilon}$ converge weakly in $\mathrm{L}^{2}\left((0, T) \times \mathbb{R}^{3}\right)$ to $\rho, J, n$ and $u$ respectively. The moment 
equations read

$$
\left\{\begin{array}{l}
\partial_{t} \rho_{\varepsilon}+\operatorname{div}_{x} J_{\varepsilon}=0 \\
\varepsilon \partial_{t} J_{\varepsilon}+\operatorname{Div}_{x} \mathbb{P}_{\varepsilon}+\sqrt{\varepsilon} \rho_{\varepsilon} \nabla_{x} \Phi=-J_{\varepsilon}+u_{\varepsilon}-\nabla_{x} \Phi \\
\quad=\sqrt{\varepsilon}\left(\sqrt{\varepsilon} \partial_{t} J_{\varepsilon}+\operatorname{Div}_{x} \mathbb{R}_{\varepsilon}+\rho_{\varepsilon} \nabla_{x} \Phi\right)+\nabla_{x} \rho_{\varepsilon} .
\end{array}\right.
$$

Hence, letting $\varepsilon \rightarrow 0$ yields

$$
-J+u-\nabla_{x} \Phi=\nabla_{x} \rho .
$$

Accordingly, we deduce that the limit satisfies $\nabla_{x} \rho+\nabla_{x} \Phi \in \mathrm{L}^{2}\left((0, T) \times \mathbb{R}^{3}\right)$. Furthermore, the equations for $\partial_{t} \rho_{\varepsilon}, \partial_{t} n_{\varepsilon}$ and $\partial_{t} u_{\varepsilon}$ provide compactness in $\mathrm{C}_{w}\left([0, T], \mathrm{L}^{2}\left(\mathbb{R}^{3}\right)\right)$.

Theorem 6. Let the assumptions of Lemma 2 be fulfilled. Then, up to a subsequence, $\rho_{\varepsilon}, n_{\varepsilon}, u_{\varepsilon}$ converge in $\mathrm{C}_{w}\left([0, T], \mathrm{L}^{2}\left(\mathbb{R}^{3}\right)\right)$ to $\rho, n$, u respectively, where the limits satisfy

$$
\left\{\begin{array}{l}
\partial_{t} \rho+\operatorname{div}_{x}\left(u-\nabla_{x} \Phi\right)-\Delta_{x} \rho=0, \\
\partial_{t} n+\bar{n} \operatorname{div}_{x} u=0, \\
\bar{n} \partial_{t} u+p^{\prime}(\bar{n}) \nabla_{x} n+\left(1+\alpha^{\prime} \bar{n}\right) \nabla_{x} \Phi+\nabla_{x} \rho=0,
\end{array}\right.
$$

with initial data $(\rho, n, u)_{t=0}=\lim _{\varepsilon \rightarrow 0}\left(\rho_{\varepsilon, 0}, n_{\varepsilon, 0}, u_{\varepsilon, 0}\right)$ weakly in $\mathrm{L}^{2}\left(\mathbb{R}^{3}\right)$.

Corollary 2. Under the same assumptions, $\rho_{\varepsilon}$ converges to $\rho$ strongly in $\mathrm{L}_{\text {loc }}^{2}\left(\mathbb{R}^{+} \times \mathbb{R}^{3}\right)$. When $\operatorname{sign}(\alpha)=+1$, assuming that

$$
\lim _{|x| \rightarrow \infty} \Phi(x)=+\infty
$$

then $\rho_{\varepsilon}$ converges to $\rho$ strongly in $\mathrm{L}^{p}\left((0, T) \times \mathbb{R}^{3}\right)$, for $1 \leq p<2$. Furthermore, we have

$$
\lim _{\varepsilon \rightarrow 0} \int_{0}^{T} \int_{\omega} \int_{\mathbb{R}^{3}}\left|f_{\varepsilon}-\rho M(\xi)\right| \mathrm{d} \xi \mathrm{d} x \mathrm{~d} t=0,
$$

where $\omega$ is any bounded set or $\omega=\mathbb{R}^{3}$ when $\operatorname{sign}(\alpha)=+1$ and (6.7) holds.

Proof. We obtain the strong convergence of the macroscopic density by using a compensated compactness argument. This argument first appeared for diffusion asymptotics of hyperbolic problems in [38]; then, it has been applied in the framework of kinetic equations [37,30]. First, we write (6.5) as $\operatorname{div}_{t, x}\left(\rho_{\varepsilon}, J_{\varepsilon}\right)=0$, and

$$
\nabla_{x} \rho_{\varepsilon}=\sqrt{\varepsilon}\left(-\sqrt{\varepsilon} \partial_{t} J_{\varepsilon}-\operatorname{Div}_{x} \mathbb{R}_{\varepsilon}\right)+u_{\varepsilon}-J_{\varepsilon},
$$

respectively. Therefore, the curl (with respect to $(t, x)$ variables) of the vector field $\left(\rho_{\varepsilon}, 0,0,0\right) \in \mathbb{R}^{4}$ belongs to a compact set of $\mathrm{H}^{-1}((0, T) \times B(0, R))$ for any $0<T, R<\infty$. Then a direct application of the Div-Curl lemma [44] tells us that $\left(\rho_{\varepsilon}, J_{\varepsilon}\right) \cdot\left(\rho_{\varepsilon}, 0\right)=\rho_{\varepsilon}^{2} \rightarrow(\rho, J) \cdot(\rho, 0)=\rho^{2}$ in $\mathcal{D}^{\prime}\left(\mathbb{R}^{+} \times \mathbb{R}^{3}\right)$. 
This proves the strong convergence of $\rho_{\varepsilon}$ to $\rho$ in $\mathrm{L}_{\text {loc }}^{2}\left(\mathbb{R}^{+} \times \mathbb{R}^{3}\right)$. When $(6.7)$ holds, we have furthermore

$$
\lim _{A \rightarrow \infty}\left(\sup _{\varepsilon>0, t \in(0, T)}\left\{\int_{|x| \geq R} \rho_{\varepsilon}(t, x) \mathrm{d} x+\int_{|x| \geq A} \rho(t, x) \mathrm{d} x\right\}\right)=0 .
$$

This allows to obtain the strong convergence globally in $\mathrm{L}^{1}\left((0, T) \times \mathbb{R}^{3}\right)$, and then in $\mathrm{L}^{p}\left((0, T) \times \mathbb{R}^{3}\right), 1 \leq p<2$.

Eventually, we split as follows

$$
\begin{aligned}
& \int_{0}^{T} \int_{\omega} \int_{\mathbb{R}^{3}}\left|f_{\varepsilon}-\rho M(\xi)\right| \mathrm{d} \xi \mathrm{d} x \mathrm{~d} t \\
& \quad \leq \int_{0}^{T} \int_{\omega} \int_{\mathbb{R}^{3}}\left|f_{\varepsilon}-\rho_{\varepsilon} M(\xi)\right| \mathrm{d} \xi \mathrm{d} x \mathrm{~d} t+\int_{0}^{T} \int_{\omega}\left|\rho-\rho_{\varepsilon}\right| \mathrm{d} x \mathrm{~d} t .
\end{aligned}
$$

We have just shown that the second integral in the right hand side tends to 0 as $\varepsilon \rightarrow 0$. Then, in the case $\omega=\mathbb{R}^{3},(6.7)$ together with Lemma 2 imply that

$$
\lim _{A \rightarrow \infty}\left(\sup _{\varepsilon>0} \int_{0}^{T} \int_{|x|>A} \int_{\mathbb{R}^{3}}\left(f_{\varepsilon}+\rho_{\varepsilon} M(\xi)\right) \mathrm{d} \xi \mathrm{d} x \mathrm{~d} t\right)=0 .
$$

Therefore, it only remains to study the integral over the bounded set $\omega=$ $B(0, A)$. By using the Sobolev inequality [3, Corollary 2.18] we obtain for some $\Lambda>0$

$$
\begin{aligned}
\int_{\mathbb{R}^{3}}\left|f_{\varepsilon}-\rho_{\varepsilon} M(\xi)\right| \mathrm{d} \xi & \leq\left(\int_{\mathbb{R}^{3}}\left|f_{\varepsilon}-\rho_{\varepsilon} M(\xi)\right|^{2} \frac{1}{M(\xi)} \mathrm{d} \xi\right)^{1 / 2} \\
& \leq \rho_{\varepsilon}\left(\int_{\mathbb{R}^{3}}\left|\frac{f_{\varepsilon}}{\rho_{\varepsilon} M(\xi)}-1\right|^{2} M(\xi) \mathrm{d} \xi\right)^{1 / 2} \\
& \leq \rho_{\varepsilon} \Lambda\left(\int_{\mathbb{R}^{3}}\left|\nabla_{\xi}\left(\frac{f_{\varepsilon}}{\rho_{\varepsilon} M(\xi)}\right)\right|^{2} M(\xi) \mathrm{d} \xi\right)^{1 / 2} \\
& \leq \Lambda\left(\int_{\mathbb{R}^{3}}\left|\nabla_{\xi}\left(\frac{f_{\varepsilon}}{M(\xi)}\right)\right|^{2} M(\xi) \mathrm{d} \xi\right)^{1 / 2} .
\end{aligned}
$$

The integral of the right hand side over $(0, T) \times \omega$ is dominated by

$$
\begin{aligned}
& \sqrt{T|\omega|}\left(\int_{0}^{T} \int_{\mathbb{R}^{3}} \int_{\mathbb{R}^{3}}\left|\nabla_{\xi}\left(\frac{f_{\varepsilon}}{M(\xi)}\right)\right|^{2} M(\xi) \mathrm{d} \xi \mathrm{d} x \mathrm{~d} t\right)^{1 / 2} \\
& \leq \sqrt{2 T|\omega|}\left(\int_{0}^{T} \int_{\mathbb{R}^{3}} \int_{\mathbb{R}^{3}}\left(\left|D_{\varepsilon}\right|^{2}+\varepsilon\left|u_{\varepsilon}\right|^{2} M(\xi)\right) \mathrm{d} \xi \mathrm{d} x \mathrm{~d} t\right)^{1 / 2} \\
& \leq C \sqrt{\varepsilon} .
\end{aligned}
$$

This ends the proof. 
Remark 11. By uniqueness of the solution of the limit system, if the initial data converge, then, the statement holds for the entire sequence.

\section{REFERENCES}

[1] Amsden A. A., Kiva-3V Release 2, Improvements to Kiva-3V, Tech. Rep., Los Alamos National Laboratory (1999).

[2] Amsden A. A., O'Rourke P. J., Butler T. D., Kiva-2, a computer program for chemical reactive flows with sprays, Tech. Rep., Los Alamos National Laboratory (1989).

[3] Arnold A., Markowich P., Toscani G., Unterreiter A., On convex Sobolev inequalities and the rate of convergence to equilibrium for Fokker-Plan ck type equations, Comm. Partial Differential Equations 26 (2001) 43-100.

[4] Arnold A., Markowich P., Toscani G., Unterreiter A., On generalized CsiszrKullback inequalities, Monatshefte für Mathematik 131, No.3, (2000) 235-253.

[5] Baranger C., Modélisation, étude mathématique et simulati on des collisions dans les fluides complexes, Thèse ENS Cachan, Juin 2004.

[6] Baranger C., Baudin G., Boudin L., Després B., Lagoutière F., Lapébie E. TAKAhashi T., Liquid jet generation and break-up, in Numerical Methods for Hyperbolic and Kinetic Equations, S. Cordier, Th. Goudon, M. Gutnic, E. Sonnendrucker Eds., IRMA Lectures in Mathematics and Theoretical Physics (EMS Publ. House) to appear.

[7] Bardos C., Santos R., Sentis R., Diffusion approximation and computation of the critical size, Trans. Amer. Math. Soc. 284 (1984) 617-649.

[8] Beals R., Protopopescu V., Abstract time-dependent transport equations, J. Math. Anal. Appl. 121 (1987) 370-405.

[9] Benedetto D., Caglioti E., Golse F., Pulvirenti M., A hydrodynamic model arising in the context of granular media, Comput. Math. Appl. 38 (1999) 121-131.

[10] Berthonnaud P., Limites fluides pour des modèles cinétiques de brouillards de gouttes monodispersés, C. R. Acad. Sci., 331 (2000) 651-654.

[11] Berres S., Bürger R., Karlsen K. H., Tory E. M., Strongly degenerate parabolichyperbolic systems modeling polydisperse sedimentation with compression, SIAM J. Appl. Math. 64 (2003) 41-80.

[12] Cáceres M. J., Carrillo J. A., Dolbeault J., Nonlinear stability in $L^{p}$ for a confined system of charged particles, SIAM J. Math. Anal. 34 (2002) 478-494.

[13] Caflisch R., Papanicolaou G., Dynamic theory of suspensions with Brow nian effects, SIAM J. Appl. Math. 43 (1983) 885-906.

[14] Carrillo J. A., Jüngel A., Markowich P. A., Toscani G., Unterreiter A. Entropy dissipation methods for degenerate parabolic systems and generalized Sobolev inequalities, Monatshefte für Mathematik 133 (2001) 1-82.

[15] Carrillo, J. A., Toscani, G., Asymptotic $L^{1}$-decay of solutions of the porous medium equation to self-similarity, Indiana Univ. Math. J. 49 (2000) 113-141.

[16] Cercignani C., The Boltzmann equation and its applications, Applied Mathematical Sciences, 67 (Springer-Verlag, 1988).

[17] Cessenat M., Théorèmes de trace $L^{p}$ pour des espaces de fonctions de la neutronique, C. R. Acad. Sci. Paris Sér. I Math. 299 (1984) 831-834 and C. R. Acad. Sci. Paris Sér. I Math. 300 (1985) 89-92.

[18] DarrozÈs J.-S., Guiraud J.-P., Généralisation formelle du théorème $H$ en présence de parois, C. R. Acad. Sci. Paris, 262 (1966) 369-371. 
[19] Dautray R., Lions J.-L., Analyse mathématique et calcul numérique pour les sciences et les techniques, Tome 3, Collection du Commissariat à l'Energie Atomique: Série Scientifique (Masson, 1985).

[20] Dolbeault J., Free energy and solutions of the Vlasov-Poisson-Fokker-Planck system: external potential and confinement (large time behavior and steady states), J. Math. Pures Appl. (9) 78 (1999) 121-157.

[21] Dolbeault J., Rein G., Time-dependent rescalings and Lyapunov functionals for the Vlasov-Poisson and Euler-Poisson systems, and for related models of kinetic equations, fluid dynamics and quantum physics, Math. Models Methods Appl. Sci. 11 (2001) 407-432.

[22] Domelevo K., Well-posedness of a kinetic model of dispersed two-phase flow with point-particles and stability of travelling waves, Discrete Contin. Dyn. Syst. Ser. B 2 (2002) 591-607.

[23] Domelevo K., Roquejoffre J. M., Existence and stability of traveling wave solutions in a kinetic model of two-phase flows, Comm. PDE 24 (1999) 61-108.

[24] Domelevo K., Vignal M.-H., Limites visqueuses pour des systèmes de type FokkerPlanck-Burgers unidimensionnels, C. R. Acad. Sci. 332 (2001) 863-868.

[25] Einstein A., On the motion of small particles suspended in liquids at rest required by the molecular-kinetic theory of heat, Annalen der Physik 17 (1905) 549-560.

[26] Gavrilyuck S., Teshukhov V., Kinetic model for the motion of compress ible bubbles in a perfect fluid, Eur. J. Mech. B/Fluids 21 (2002) 469-491.

[27] Goudon T., Asymptotic problems for a kinetic model of two-phase flow, Proc. Royal Soc. Edimburgh. 131 (2001) 1371-1384.

[28] Goudon T., Jabin P.-E., Vasseur A., Hydrodynamic limits for the Vlasov-NavierStokes equations: Light particles regime, Indiana University Math. J. 53 (2004) 14951516.

[29] Goudon T., Jabin P.-E., VAsseur A., Hydrodynamic limits for the Vlaso v-NavierStokes equations: Fine particles regime, Indiana University Math. J. 53 (2004) 15171536 .

[30] Goudon T., Poupaud F., Approximation by homogeneization and diffusion of kinetic equations, Comm. PDE. 26 (2001) 537-569.

[31] Hamdache K., Global existence and large time behaviour of solutions for the VlasovStokes equations, Japan J. Indust. Appl. Math. 15 (1998) 51-74.

[32] Hamdache K., Unpublished work, Personal Communication.

[33] Herrero H., Lucquin-Desreux B., Perthame B., On the motion of dispers ed balls in a potential flow: a kinetic description of the added mass effect, SIAM J. Appl. Math. 60 (1999) 61-83.

[34] JABIN P. E., Large time concentrations for solutions to kinetic equations with energy dissipation, Comm. PDE. 25 (2000) 541-557.

[35] JABIN P. E., Macroscopic limit of Vlasov type equations with friction, Ann. IHP Anal. Non Linéaire 17 (2000) 651-672.

[36] Jabin P. E., Perthame B., Notes on mathematical problems on the dynamics of dispersed particles interacting through a fluid in Modeling in applied sciences, a kinetic theory approach, N. Bellomo, M. Pulvirenti Eds. (Birkhäuser, 2000), pp. $111-147$.

[37] Lions P.-L., Toscani G., Diffusive limits for finite velocity Boltzmann kinetic models, Revista Mat. Iberoamer. 13 (1997) 473-513.

[38] Marcati P., Milani A., The one-dimensional Darcy's law as the limit of a compressible Euler flow, J. Differential Equations 84 (1990) 129-147. 
[39] Masmoudi N., Saint-Raymond L., From the Boltzmann equation to the StokesFourier system in a bounded domain, Comm. Pure Appl. Math. 56 (2003) 1263-1293.

[40] Mellet A., Vasseur A., Work in progress, Personal communication.

[41] O'Rourke P. J., Collective drop effects on vaporizing liquid sprays, PhD thesis, Los Alamos National Laboratory (1981).

[42] Rein G., Non-linear stability of gaseous stars, Arch. Rat. Mech. Anal. 168 (2003) $115-130$.

[43] Russo G., Smereka P., Kinetic theory for bubbly flows I, II, SIAM J. Appl. Math. 56 (1996) 327-371.

[44] Tartar L., Compensated compactness and applications to pde, in Herriot-Watt Symp., vol. IV, R. Knopps Eds., Res. Notes in Math, vol. 39, pp. 136-212 (Pitman, 1979).

[45] Williams F. A., Combustion theory (Benjamin Cummings Publ., 2nd ed., 1985).

[46] Williams F. A., Spray combustion and atomization, Physics of Fluids 1 (1958) $541-555$.

José A. Carrillo

ICREA and Departament de Matemàtiques,

Universitat Autònoma de Barcelona,

E-08193 Bellaterra, Spain.

E-mail: carrillo@mat.uab.es

Thierry Goudon

Labo. Paul Painlevé, UMR 8524,

C.N.R.S.-Université Sciences et Technologies de Lille,

Cité Scientifique, F-59655 Villeneuve d'Ascq cedex, France.

E-mail: Thierry.Goudon@math.univ-lille1.fr 